\title{
Dory-Guest-Harris instability as a benchmark for continuum kinetic Vlasov-Poisson simulations of magnetized plasmas
}

\author{
G. V. Vogman ${ }^{\mathrm{a}}$, P. Colella ${ }^{\mathrm{b}}$, U. Shumlak ${ }^{\mathrm{c}}$, \\ ${ }^{a}$ Applied Science 85 Technology Program, University of California, Berkeley, CA 94720, USA \\ ${ }^{b}$ Computational Research Division, Lawrence Berkeley National Laboratory, Berkeley, CA 94720, USA \\ and Department of Electrical Engineering 86 Computer Science, University of California, Berkeley, CA \\ 94720, USA \\ ${ }^{c}$ Aerospace 8 Energetics Research Program, University of Washington, Seattle, WA 98195-2250, USA
}

\begin{abstract}
The Dory-Guest-Harris instability is demonstrated to be a well-suited benchmark for continuum kinetic Vlasov-Poisson algorithms. The instability is a special case of perpendicularlypropagating kinetic electrostatic waves in a warm uniformly magnetized plasma. A complete derivation of the closed-form linear theory dispersion relation for the instability is presented. The electric field growth rates and oscillation frequencies specified by the dispersion relation provide concrete measures against which simulation results can be quantitatively compared. A fourth-order continuum kinetic algorithm is benchmarked against the instability, and is demonstrated to have good convergence properties and close agreement with theoretical growth rate and oscillation frequency predictions. Second-order accurate simulations are also shown to be consistent with theoretical predictions, but require higher resolution for convergence. The Dory-Guest-Harris instability benchmark extends the scope of current standard test problems by providing a substantive means of validating continuum kinetic simulations of magnetized plasmas in higher-dimensional 3D $\left(x, v_{x}, v_{y}\right)$ phase space. The linear theory analysis, initial conditions, algorithm description, and comparisons between theoretical predictions and simulation results are presented.
\end{abstract}

Keywords: Vlasov-Poisson, Dory-Guest-Harris instability, plasma kinetic theory, continuum kinetic benchmark, electrostatic waves in magnetized plasma, phase space

\section{Introduction}

Kinetic theory of plasmas treats each particle species as a probability distribution function in six-dimensional position-velocity phase space. The evolution of the distribution function is governed by the Boltzmann equation coupled to Maxwell's equations. When collisions can be neglected, the Boltzmann equation is reduced to the Vlasov equation.

These governing equations are often solved using Particle-in-Cell (PIC) methods. PIC methods sample a phase space distribution function with superparticles, and advance these superparticles in up to three spatial dimensions using the Lorentz force, which relates particle motion to fields [1]. In contrast, continuum methods - also known as Eulerian methods - 
evolve the full kinetic distribution function by solving the Boltzmann-Maxwell equation system in up to six dimensions of phase space. The computational cost of continuum methods scales geometrically with the number of dimensions thereby making full phase space continuum methods computationally costly. Recent advances in supercomputing capability and hyperbolic partial differential equation solvers, however, have made continuum methods a viable alternative to PIC. Examples include the development of high-order moment-conserving Vlasov-Poisson solvers in 2D $\left(x, v_{x}\right)[2,3,4,5,6,7]$, relativistic and non-relativistic VlasovMaxwell solvers in 3D $\left(x, v_{x}, v_{y}\right)[8,9]$ and in $4 \mathrm{D}\left(x, y, v_{x}, v_{y}\right)[10,11]$, five-dimensional continuum gyrokinetics algorithms for strong guide-field physics [12,13], and various continuum kinetic methods for collisional physics modeled by the Boltzmann equation [14].

Continuum methods offer a number of advantages. They can be cast in conservation law form so as to preserve velocity moments of the distribution function i.e. conserve mass, momentum, and energy. They also allow for the use of high order methods, which enhance the temporal and spatial accuracy of the solution. Unlike PIC methods, the continuum approach is not subject to noise $[15,16,17]$ and thus can retain accuracy over longer periods of simulated time. In terms of computational efficiency, parallelization for continuum methods is achieved through static domain decomposition, which is especially conducive to scalable load balancing on supercomputers. Continuum method scalability is also facilitated by the fact that they involve more arithmetic operations per byte of data, thus ensuring that parallelization is dominated by computation, not communication [18]. This still holds even when dynamic domain decomposition is used, e.g. in numerical methods that employ adaptive mesh refinement techniques.

Kinetic model algorithms in general, and continuum methods in particular, lack an arsenal of standardized benchmarks problems. This is in part due to the comparatively recent development of continuum methods, but more fundamentally because PIC and continuum methods have different limitations. Whereas PIC accuracy is limited by both the number of particles and grid resolution in up to three spatial dimensions, continuum method accuracy is limited by grid resolution in up to six phase space dimensions. A consequence of this is that PIC methods most effectively model cold plasmas and beams, whereas continuum methods are more conducive to modeling finite or high temperature phenomena. Thus standard benchmarks for PIC involving cold plasma assumptions with delta function initial conditions in velocity space [1] do not in general carry over to continuum methods.

Existing standardized benchmarks for continuum methods are largely limited to electrostatic phenomena that can be simulated in $2 \mathrm{D}\left(x, v_{x}\right)$ phase space. These standardized test problems include: weak Landau damping [2, 3, 5, 6, 7, 19, 20], strong Landau damping $[3,4,5,6,7,19]$, two-stream instability [3, 4, 5, 6, 7, 19], bump-on-tail instability [2, 3], and Langmuir waves $[10,21]$. In addition, ion-acoustic turbulence has been used as a qualitative benchmark [2]. Plasma in the presence of magnetic fields have been simulated with continuum methods using the following much less developed test problems: particle gyration $[8,10]$, high-frequency electromagnetic waves [10], Raman scattering in 2D $\left(x, v_{x}\right)$ [22] and in $4 \mathrm{D}\left(x, y, v_{x}, v_{y}\right)$ [10], Weibel instability [8, 23, 24, 25], Bernstein waves [20], relativistic wakefield acceleration [8, 9], and the Kelvin-Helmholtz instability [11, 26, 27].

To address the paucity of benchmark problems for continuum methods, this paper in- 
vestigates the Dory-Guest-Harris instability [28], which is a type of cyclotron harmonic instability [29, 30, 31] that is closely related to Bernstein modes [32], as a means of validating continuum kinetic simulations. In particular, the instability provides a straightforward means by which to benchmark simulations of magnetized plasmas in a limited number of dimensions: one spatial dimension and two velocity dimensions (1D2V). The paper is organized such that Sec. 2 draws on the theory of perpendicularly-propagating electrostatic waves in a warm magnetized plasma and provides a derivation of a closed-form dispersion relation for multiple species with arbitrary equilibrium distribution functions. Section 3 explores the dispersion relation characteristics for a Dory-Guest-Harris distribution and presents benchmarks against which continuum kinetic methods can be tested. Section 4 outlines a continuum fourth-order accurate 3D $\left(x, v_{x}, v_{y}\right)$ Vlasov-Poisson solver that is used to simulate the instability. Sections 5 and 6 present simulation results and discuss the utility of the theoretical benchmarks as a validation platform.

\section{Linear analysis of electrostatic $k_{\|}=0$ waves in uniformly magnetized plasma}

In kinetic theory constituent particle species in a plasma are described by probability distribution functions in phase space. The probability distribution function $f_{s}(\mathbf{x}, \mathbf{v}, t)$ for species $s$ depends on the position coordinate $\mathbf{x}$, velocity coordinate $\mathbf{v}$, and time $t$. The evolution of the distribution function in the case of electrostatic fields is described by the Vlasov equation and Gauss's law. The non-dimensional form of this system of governing equations is

$$
\begin{aligned}
0 & =\frac{\partial f_{s}}{\partial t}+\mathbf{v} \cdot \frac{\partial f_{s}}{\partial \mathbf{x}}+\frac{Z_{s}}{M_{s}}\left(\mathbf{E} \cdot \frac{\partial f_{s}}{\partial \mathbf{v}}+\frac{\omega_{c}}{\omega_{p}} \mathbf{v} \times \hat{\mathbf{b}} \cdot \frac{\partial f_{s}}{\partial \mathbf{v}}\right) \\
\nabla \cdot \mathbf{E} & =\sum_{s} Z_{s} \int f_{s} d \mathbf{v}
\end{aligned}
$$

where $f$ is the non-dimensional distribution function normalized by a nominal electron density $n_{0} ; \hat{\mathbf{b}}$ is the direction of the magnetic field $\mathbf{B}=B \hat{\mathbf{b}} ; M_{s}=m_{s} / m_{e}$ is the ratio of the mass of species $s$ to the electron mass; and $Z_{s}=q_{s} /|e|$ is ionization state i.e. the ratio of the charge of species $s$ to the electron charge. Note that $t$ is the non-dimensional time, i.e. the physical time normalized by the electron plasma frequency. The factor in front of the cross product term is the ratio of electron cyclotron frequency $\omega_{c}=e B / m_{e}$ to electron plasma frequency $\omega_{p}=\left(n_{0} e^{2} / \varepsilon_{0} m_{e}\right)^{1 / 2}$, where $-e$ is the electron charge and $\varepsilon_{0}$ is the permittivity of free space. For electrostatic waves the electric field can be defined in terms of the electric potential $\Phi$, such that $\mathbf{E}=-\nabla \Phi$. Thus Gauss's law can be expressed as Poisson's equation,

$$
-\nabla^{2} \Phi=\sum_{s} Z_{s} \int f_{s} d \mathbf{v} .
$$

The properties of small-amplitude waves depend on the equilibrium fields $\left(\mathbf{E}_{0}, \mathbf{B}_{0}\right)$ and the equilibrium probability distribution function $f_{s 0}(\mathbf{x}, \mathbf{v})$. The response of equilibria to perturbations can be analyzed using the linearized governing equations. In the linearization 
procedure the fields and the distribution function $f_{s}(\mathbf{x}, \mathbf{v}, t)$ are expressed as a sum of an equilibrium part and a perturbation about that equilibrium [33]: $\mathbf{E}=\mathbf{E}_{0}+\mathbf{E}_{1}, \mathbf{B}=\mathbf{B}_{0}+\mathbf{B}_{1}$, $f_{s}=f_{s 0}+f_{s 1}$. It is assumed that the perturbation values are much smaller in magnitude than the equilibrium values, e.g. $f_{s 1} \ll f_{s 0}$, such that nonlinear products of the perturbation terms can be neglected.

For an electrostatic system with a time-invariant equilibrium magnetic field $\left(\mathbf{B}=\mathbf{B}_{0}\right)$ and no equilibrium electric field $\left(\mathbf{E}=\mathbf{E}_{1}\right)$, the departure away from equilibrium is described by the linearized Vlasov equation and Gauss's law. These are expressed in their non-dimensional form as

$$
\begin{aligned}
0 & =\frac{\partial f_{s 1}}{\partial t}+\mathbf{v} \cdot \frac{\partial f_{s 1}}{\partial \mathbf{x}}+\frac{Z_{s}}{M_{s}}\left(\mathbf{E}_{1} \cdot \frac{\partial f_{s 0}}{\partial \mathbf{v}}+\frac{\omega_{c}}{\omega_{p}} \mathbf{v} \times \hat{\mathbf{b}}_{0} \cdot \frac{\partial f_{s 1}}{\partial \mathbf{v}}\right) \\
\nabla \cdot \mathbf{E}_{1} & =\sum_{s} Z_{s} \int f_{s 1} d \mathbf{v},
\end{aligned}
$$

where $f_{s 0}\left(v_{\perp}, v_{\|}\right)$is the equilibrium velocity distribution for species $s, v_{\perp}$ and $v_{\|}$are the non-dimensional components of electron velocity that are perpendicular and parallel to the equilibrium magnetic field $\mathbf{B}_{0}=B_{0} \hat{\mathbf{b}}_{0}$, and $f_{s 1}$ is the first order perturbation of the probability distribution function away from equilibrium.

Equations (4) and (5) can be used to derive a dispersion relation for small-amplitude waves. For the purposes of this paper, consideration is limited to electrostatic waves propagating perpendicular to the equilibrium magnetic field with wave vector components $k_{\perp} \neq 0$ and $k_{\|}=0$. Such waves are not subject to the effects of collisionless cyclotron or Landau damping $[28,31]$. Without loss of generality, let $\mathbf{B}_{0}=B_{0} \hat{\mathbf{z}}$ and $\mathbf{k}=k_{\perp} \hat{\mathbf{x}}$ to simplify the analysis. Taking advantage of the azimuthal symmetry associated with the equilibrium magnetic field, and following the procedure detailed in Ref. [34, pp. 341-351], a cylindrical coordinate system is introduced in which velocity is defined as

$$
\mathbf{v}=v_{\perp} \cos \theta \hat{\mathbf{x}}+v_{\perp} \sin \theta \hat{\mathbf{y}}+v_{\|} \hat{\mathbf{z}}
$$

Fourier transforms in space and time are applied to both $f_{1}$ and $\mathbf{E}_{1}$, such that the transformed versions are $\hat{f}_{1}$ and $\hat{\mathbf{E}}_{1}$, respectively. To analyze a single fixed wave number Fourier mode, $f_{1}=\hat{f}_{1} e^{i(\mathbf{k} \cdot \mathbf{x}-\omega t)}$ and $\mathbf{E}_{1}=\hat{\mathbf{E}}_{1} e^{i(\mathbf{k} \cdot \mathbf{x}-\omega t)}$ are substituted into Eq. (4), which yields

$$
0=-i \omega \hat{f}_{s 1}+i \mathbf{v} \cdot \mathbf{k} \hat{f}_{s 1}+\frac{Z_{s}}{M_{s}} \hat{\mathbf{E}}_{1} \cdot \frac{\partial f_{s 0}}{\partial \mathbf{v}}-\frac{Z_{s} \omega_{c}}{M_{s} \omega_{p}} \frac{\partial \hat{f}_{s 1}}{\partial \theta}
$$

Note that $\mathbf{v} \cdot \mathbf{k}=k_{\perp} v_{\perp} \cos \theta$ and let the non-dimensionalized species-dependent cyclotron frequency be defined as $\Omega_{c s}=Z_{s} \omega_{c} /\left(M_{s} \omega_{p}\right)$, then the transformed linearized Vlasov equation is

$$
0=i\left(\frac{\omega}{\Omega_{c s}}-\frac{k_{\perp} v_{\perp}}{\Omega_{c s}} \cos \theta\right) \hat{f}_{s 1}-\frac{\omega_{p}}{\omega_{c}} \hat{\mathbf{E}}_{1} \cdot \frac{\partial f_{s 0}}{\partial \mathbf{v}}+\frac{\partial \hat{f}_{s 1}}{\partial \theta}
$$


Equation (8) is an ordinary differential equation that can be analytically solved for $\hat{f}_{s 1}$. The solution is then substituted into the transformed Poisson equation

$$
k_{\perp}^{2} \hat{\Phi}_{1}=\sum_{s} Z_{s} \int \hat{f}_{s 1} d \mathbf{v}
$$

such that the variables $\hat{\mathbf{E}}_{1}, \hat{\Phi}_{1}$, and $f_{s 1}$ are eliminated. See Appendix A for details. The resulting dispersion relation, given by $D\left(\omega, k_{\perp}\right)=0$, where $D\left(\omega, k_{\perp}\right)$ is an implicit function that can be expressed as

$$
\begin{gathered}
D\left(\omega, k_{\perp}\right)=1+\sum_{s} M_{s} \frac{\omega_{p}^{2}}{\omega_{c}^{2}} \int_{0}^{\pi} \frac{\sin \left(\frac{\omega}{\Omega_{c s}} \tau\right)}{\sin \left(\frac{\omega}{\Omega_{c s}} \pi\right)} \sin (\tau) F_{0}(\tau+\pi) d \tau=0 \\
F_{0}(\tau)=\int_{0}^{\infty} f_{s 0}\left(v_{\perp}\right) J_{0}\left(2 \frac{k_{\perp} v_{\perp}}{\Omega_{c s}} \sin \frac{\tau}{2}\right) 2 \pi v_{\perp} d v_{\perp},
\end{gathered}
$$

where $J_{0}$ is the zeroth order Bessel function of the first kind. Note that $v_{\|}$dependence has been eliminated since it does not play a role for $k_{\|}=0$ waves. Note also that the dispersion relation is independent of the sign of $\Omega_{c s}$ because $J_{0}$ is an even function. Thus, for convenience, $\Omega_{c s}$ is henceforth defined to be positive such that $\Omega_{c s}=\left|Z_{s}\right| \omega_{c} /\left(M_{s} \omega_{p}\right)$. Equation (10) is the non-dimensional multi-species closed-form dispersion relation and is a generalization of the dimensional single-species closed-form expression presented without proof in Ref. [31]. The closed-form representation (see Appendix A for a complete derivation) does not involve infinite sums, and thus a solution $\omega\left(k_{\perp}\right)$ can be computed numerically with great accuracy for any arbitrary equilibrium distribution function.

The dispersion relation describes the propagation of waves of the form $e^{i\left(k_{\perp} x-\omega t\right)}$. To obtain an explicit relation for frequency as a function of wavenumber, i.e. $\omega\left(k_{\perp}\right)$, the roots of Eq. (10) are evaluated numerically for real values of $k_{\perp}$. In general frequency $\omega$ is complexvalued, such that $\omega=\omega_{r}+i \omega_{i}$. The real and imaginary components of frequency indicate the temporal evolution of the perturbation: $\omega_{r}$ provides the oscillation frequency and $\omega_{i}$, depending on the sign, provides the growth $\left(\omega_{i}>0\right)$ or decay $\left(\omega_{i}<0\right)$ rate. For a given mixed-complex frequency solution of the dispersion relation, its complex-conjugate is also a solution; however, because exponential growth $e^{\left|\omega_{i}\right| t}$ dominates over an exponential rate of decay $e^{-\left|\omega_{i}\right| t}$, only the exponentially growing solution is of consequence.

\section{Dory-Guest-Harris instability in warm plasmas}

The dispersion relation in Eq. (10) describes the properties of electrostatic waves propagating perpendicular to a uniform externally applied magnetic field in a spatially uniform infinite plasma. The properties of these waves over small and large time scales are relevant to a number of applications [35] including neutral beam heating in tokamaks [36, 37], electrostatic cyclotron harmonic emissions in the magnetosphere [38, 39], auroral precipitation 
[40, 41], magnetic mirror loss cones [42], and electron cyclotron resonance heating [43]. Notably, the exact dispersion relation properties are determined by the equilibrium distribution function in velocity space, $f_{0}\left(v_{\perp}\right)$.

Different probability distribution functions and their associated stability properties have been investigated theoretically $[28,30,31,32,44]$ and computationally $[29,45,46]$. In particular, Dory et al. [28] identified that such waves are unstable for a warm ring velocity distribution provided that (a) the distribution $f_{0}\left(v_{\perp}\right)$ is sufficiently peaked, (b) the ratio of plasma frequency to cyclotron frequency is above a specified threshold value, and (c) the wave number falls in a specific range. The class of single-species velocity distribution functions investigated by Dory et al. [28] can be expressed as:

$$
f_{0}\left(v_{\perp}\right)=\frac{1}{\pi \alpha_{\perp}^{2} j !}\left(\frac{v_{\perp}}{\alpha_{\perp}}\right)^{2 j} \exp \left(-\frac{v_{\perp}^{2}}{\alpha_{\perp}^{2}}\right)
$$

where $f_{0}\left(v_{\perp}\right)$ is the normalized equilibrium distribution function whose integral over the velocity domain has a value of one. Parameter $\alpha_{\perp}$ and non-negative integer $j$ control the velocity spread of the distribution and the location of the peak. The distribution is peaked at $v_{\perp 0}=j^{1 / 2} \alpha_{\perp}$ and has a half-width at half-maximum that is approximated by $\alpha_{\perp} / j^{1 / 2}$. The half-width approximation is incorrectly presented in Ref. [28].

Note the dependence of the velocity distribution function on $j: j=0$ corresponds to a Maxwellian, $j>0$ corresponds to a ring, and in the limit of $j \rightarrow \infty$ the distribution is a delta function ring with infinitesimal velocity spread. Figure 1 shows the velocity distribution function for different values of $j$. Dory et al. [28] identified that for $j=\{0,1,2\}$ the equilibrium distribution function is unconditionally stable; for $j=\{3,4,5\}$ it is conditionally stable and supports wave frequencies that are either purely real or purely imaginary; for $j \geq 6$ the distribution is conditionally stable and supports wave frequencies that have purely real, purely imaginary, or mixed complex frequencies.

While the dispersion relation of Eq. (10) applies more generally, the evolution of the Dory-Guest-Harris instability concerns particles of a single species. It is assumed that the plasma is composed of particle species that have disparate masses, e.g. ions and electrons, and thereby the interactions between species are not important [28]. Thus on time scales over which ion motion can be neglected, the only evolving distribution function is that of the electrons. Similarly, on time scales much greater than the electron plasma frequency, the only evolving distribution function is that of the ions. In effort to avoid ambiguity, $f$ is henceforth the electron distribution function and a fixed uniform ion background is assumed.

The dispersion relation for the Dory-Guest-Harris distribution can be solved numerically for frequency as a function of wave number. It is convenient to solve for the normalized frequency $\tilde{\omega}=\tilde{\omega}_{r}+i \tilde{\omega}_{i}$ as a function of the normalized wave number $\tilde{k}$, defined respectively as

$$
\begin{aligned}
\tilde{\omega} & =\omega / \Omega_{c} \\
\tilde{k} & =k_{\perp} v_{\perp 0} / \Omega_{c},
\end{aligned}
$$

where $\Omega_{c}=\omega_{c} / \omega_{p}$. As described in Sec. 2, time is normalized by $\omega_{p}$. Solutions to the dispersion relation are found by selecting a particular real wave number value, $\tilde{k}^{*}$, and 


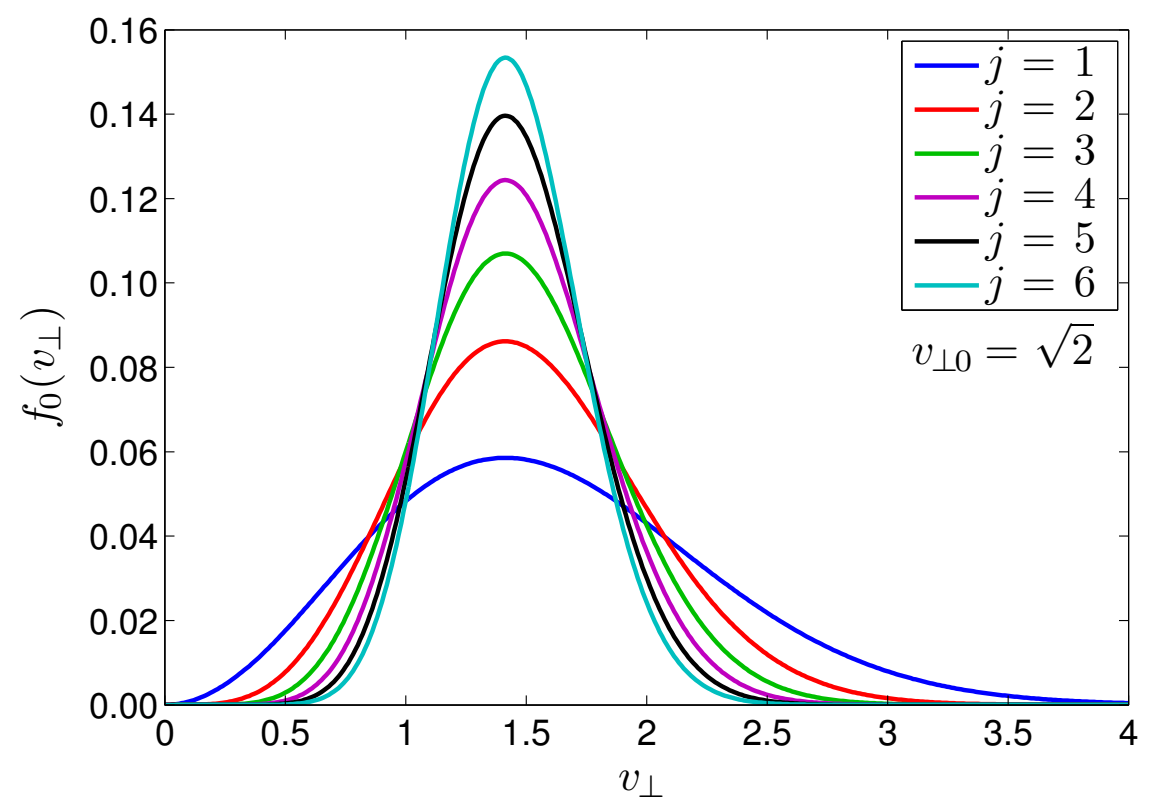

Figure 1: Dory-Guest-Harris equilibrium probability distribution function in velocity space for different values of $j$. See Eq. (12). The larger the value of non-negative integer $j$, the more peaked the distribution. When superimposed with a spatially-dependent perturbation, the distribution function is unconditionally stable for $j=\{0,1,2\}$ and conditionally stable for $j \geq 3$. 
finding the value of frequency $\tilde{\omega}$ for which the equality $D\left(\tilde{\omega}, \tilde{k}^{*}\right)=0$ is satisfied. In cases where $\tilde{\omega}$ is purely real, or purely imaginary, the bisection method can be used to find the frequency. When frequency is mixed complex, a minimization of $\left(D\left(\tilde{\omega}, \tilde{k}^{*}\right)\right)^{2}$ is performed to solve for the real and imaginary components of frequency. In the minimization method, an initial guess is informed by first evaluating $D\left(\tilde{\omega}_{r}, \tilde{\omega}_{i}, \tilde{k}^{*}\right)$ for a range of real and imaginary frequency values and then finding the intersection of the $\operatorname{Re}\left(D\left(\tilde{\omega}_{r}, \tilde{\omega}_{i}, \tilde{k}^{*}\right)\right)=0$ contours with the $\operatorname{Im}\left(D\left(\tilde{\omega}_{r}, \tilde{\omega}_{i}, \tilde{k}^{*}\right)\right)=0$ contours in the $\tilde{\omega}_{r}-\tilde{\omega}_{i}$ plane. Note that $\tilde{\omega}(\tilde{k})$ is multivalued, so that for each value of $\tilde{k}$, there are multiple values of $\tilde{\omega}$ that satisfy the dispersion relation of Eq. (10). These multiple values correspond to different harmonics. Thus, when numerically solving for frequency as a function of wavenumber, it is helpful to limit the range of $\tilde{\omega}$ over which solutions are sought.

The resulting dispersion diagram for the case of a Maxwellian distribution $(j=0$, $\alpha_{\perp}=2^{1 / 2}$ ) is shown in Fig. 2, and is consistent with the results presented in Refs. [30, 31]. Because frequency is real-valued for all values of wave number independent of the ratio $\omega_{p} / \omega_{c}$, the Maxwellian distribution is unconditionally stable. The dispersion diagrams for the unconditionally stable case of $j=2$ are shown in Fig. 3 for different values of $\omega_{p} / \omega_{c}$. As is consistent with [31], the cutoff frequencies (for which $\tilde{k}=0$ ) are: at integer multiples of the non-dimensional cyclotron frequency, $n \Omega_{c}$ with $|n|>1$; and at the non-dimensional upper hybrid frequency, $\left(1+\Omega_{c}^{2}\right)^{1 / 2}$. Likewise, the resonance frequencies (for which $\tilde{k}$ is infinite), are at $n \Omega_{c}$ with $|n|>0$.

For conditionally stable cases of $j=4$ and $j=6$ dispersion diagrams are shown in Fig. 4 and Fig. 5, respectively. Instability for the $j=4$ equilibrium distribution function is evidenced when $\tilde{\omega}_{i}>0$, which occurs for a finite range of wave numbers provided that the ratio of electron plasma frequency to cyclotron frequency exceeds the threshold value of $\approx 10.5$. In this case there are no mixed-complex frequency solutions to the dispersion

relation. For $j=6$ the distribution function is unstable for certain ranges of $\tilde{k}$ provided that $\omega_{p} / \omega_{c} \gtrsim 7.0$. Above this threshold value, instabilities with purely imaginary or mixed complex frequencies can occur. Note that the threshold value of $\omega_{p} / \omega_{c}$ above which instability can occur is smaller for larger values of $j$. These instability properties are consistent with the thresholds presented in Ref. [28]. As $j$ is increased, the $\tilde{\omega}(\tilde{k})$ for the warm ring smoothly approaches the dispersion relation for the delta function ring [28]. Dispersion diagrams for delta function distributions are presented in Refs. [1, 31].

\section{Vlasov-Poisson model and continuum algorithm}

\subsection{Non-dimensional $3 D\left(x, v_{x}, v_{y}\right)$ Vlasov-Poisson system and domain specifications}

The evolution of the Dory-Guest-Harris probability distribution function in response to electrostatic waves propagating perpendicular to an equilibrium magnetic field can be simulated in three-dimensional phase space: position coordinate $x$ and velocity coordinates $v_{x}$ and $v_{y}$, with magnetic field in the $\hat{z}$ direction and electric field of the wave in the $\hat{x}$ direction. This evolution is described by the non-relativistic Vlasov-Poisson equation system. In non-dimensional conservation law form the 3D $\left(x, v_{x}, v_{y}\right)$ Vlasov-Poisson system of governing 


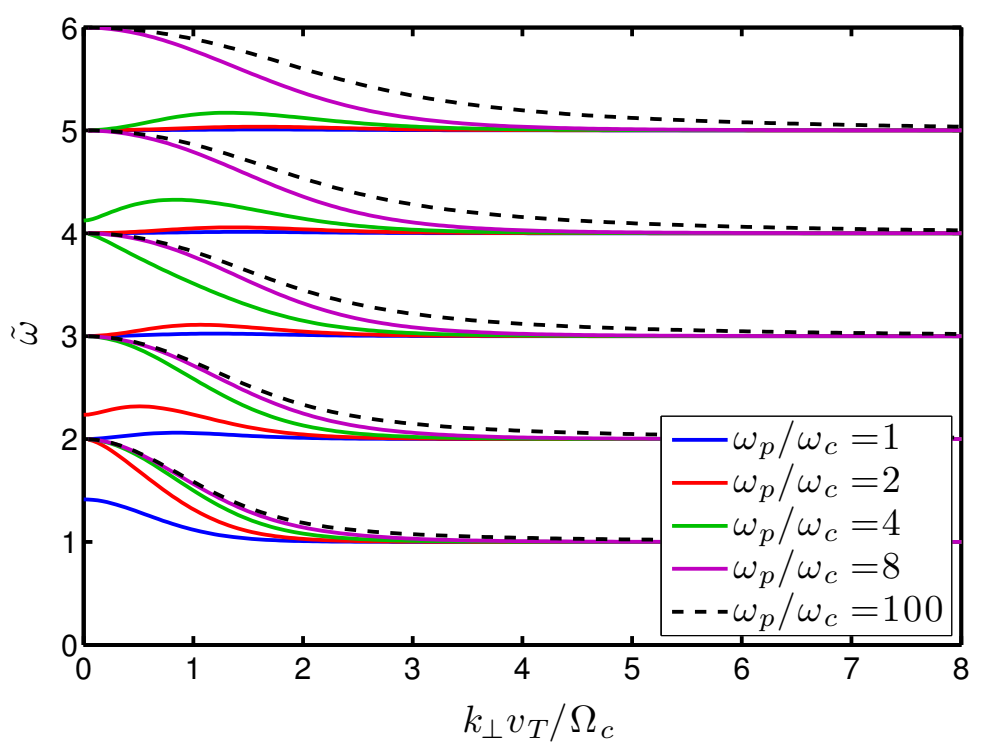

Figure 2: Dispersion diagram for a Maxwellian distribution function in velocity space, with $j=0$ and $\alpha_{\perp}=2^{1 / 2}$ in Eq. (12). The Maxwellian distribution is unconditionally stable such that frequency is real-valued, independent of $\omega_{p} / \omega_{c}$ and of $k_{\perp} v_{T} / \Omega_{c}$. Note that the wave number (see Eq. (14)) is normalized using thermal velocity defined by $v_{T}^{2}=\alpha_{\perp}^{2} / 2$ since $v_{\perp 0}$ is zero for a Maxwellian distribution.

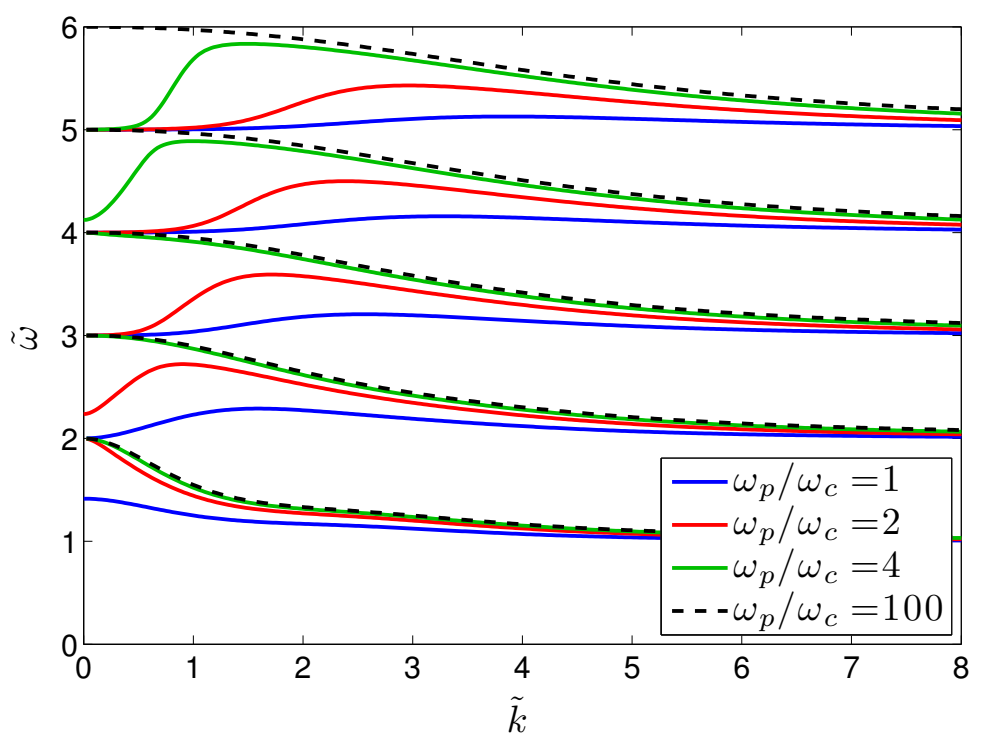

Figure 3: Dispersion diagram for an unconditionally stable warm ring velocity distribution function with $j=2$ and $v_{\perp 0}=\sqrt{2}$, namely $\tilde{\omega}_{i}=0$. Stability is evidenced by a real-valued frequency $\tilde{\omega}$ for each $\tilde{k}$, independent of the value of $\omega_{p} / \omega_{c}$. 

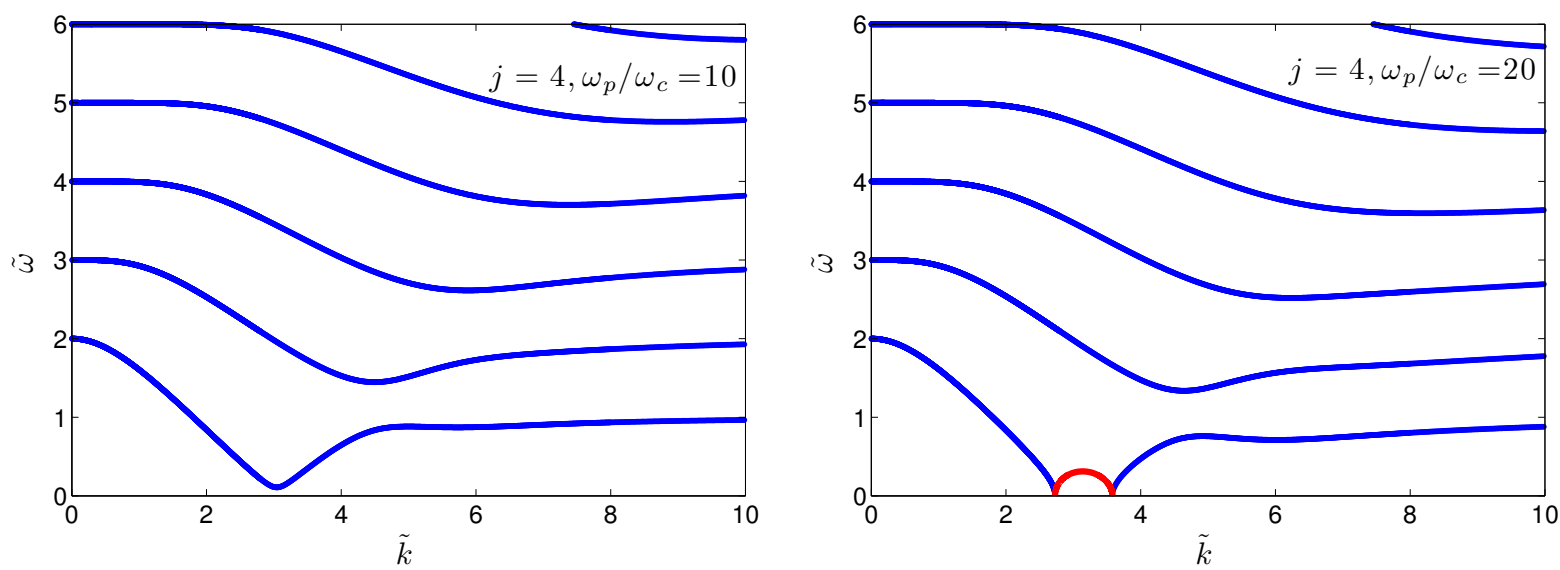

Figure 4: Dispersion diagrams for a conditionally stable velocity distribution function with $j=4$ and $v_{\perp 0}=\sqrt{2}$. For these parameters frequency is either purely real (blue) or purely imaginary (red). Instability can occur when $\omega_{p} / \omega_{c} \gtrsim 10.5$. For $\omega_{p} / \omega_{c}=20$, instability occurs when $\tilde{k} \in[2.73,3.58]$.
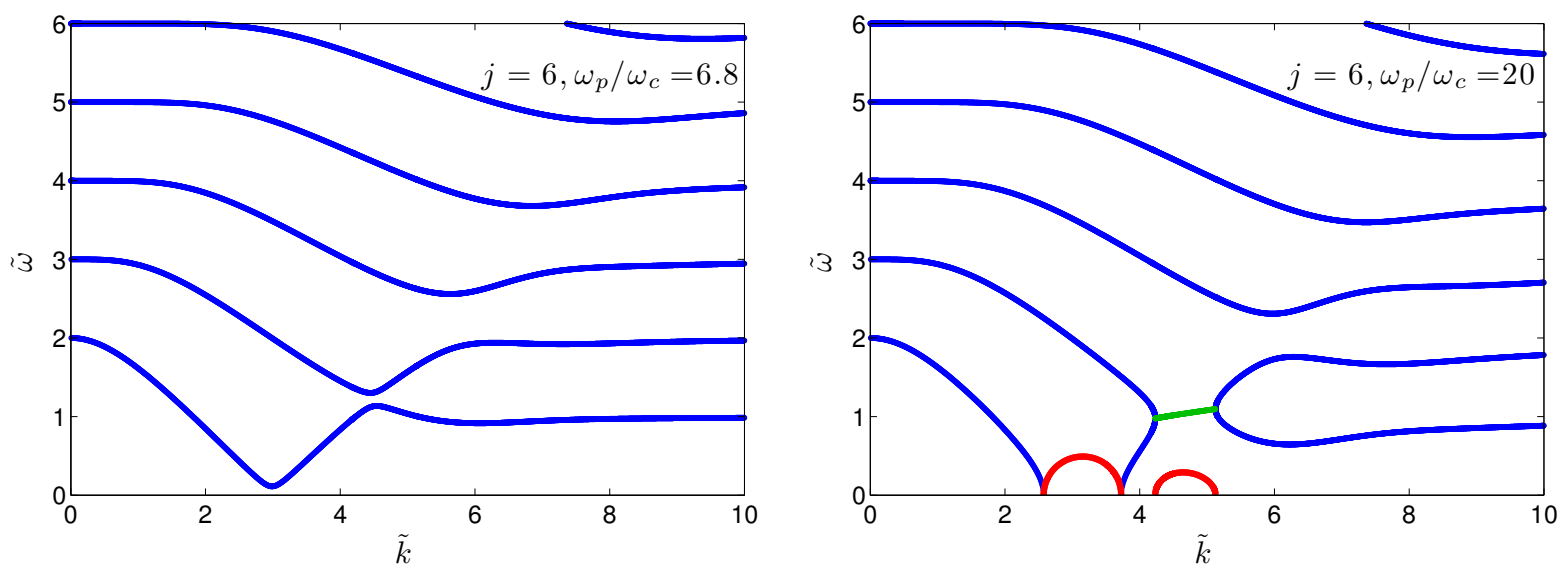

Figure 5: Dispersion diagrams for a conditionally stable velocity distribution function with $j=6$ and $v_{\perp 0}=\sqrt{2}$. Instability can occur when $\omega_{p} / \omega_{c} \gtrsim 7.0$. Real values of frequencies are shown in blue, imaginary components of frequency are shown in red, and real components of mixed-complex frequencies are shown in green. For $\omega_{p} / \omega_{c}=20$ an electrostatic wave exhibits pure growth when $\tilde{k} \in[2.57,3.73]$, and growth with propagation when $\tilde{k} \in[4.23,5.13]$. 
equations is:

$$
\begin{aligned}
0 & =\frac{\partial f}{\partial t}+\frac{\partial}{\partial x}\left(v_{x} f\right)+\frac{Z_{e}}{M_{e}} \frac{\partial}{\partial v_{x}}\left(\left[E_{x}+v_{y} \frac{\omega_{c}}{\omega_{p}}\right] f\right)+\frac{Z_{e}}{M_{e}} \frac{\partial}{\partial v_{y}}\left(-v_{x} \frac{\omega_{c}}{\omega_{p}} f\right) \\
-\frac{\partial^{2} \phi}{\partial x^{2}} & =1-\int_{-\infty}^{\infty} \int_{-\infty}^{\infty} f d v_{x} d v_{y}
\end{aligned}
$$

where Eq. (15) is the reduced dimension version of Eq. (1), $f$ is the electron distribution function, and $Z_{e} / M_{e}=-1$. The spatial domain has length $L$, such that $x \in[0, L]$. To minimize computational cost, the velocity domain is truncated such that $v_{x}, v_{y} \in\left[-v_{\max }, v_{\max }\right]$ with finite $v_{\max }$. Note that the Poisson equation contains number density contributions from the electrons and from the ions, which are assumed to be fixed and uniformly distributed in space. The system is quasineutral such that the righthand side of Eq. (16) integrated over the spatial domain has a value of zero.

The domain is divided into $N_{x} \times N_{v_{x}} \times N_{v_{y}}$ cells where $N_{x}, N_{v_{x}}$, and $N_{v_{y}}$ are the number of cells in each of the three phase space directions. Boundary conditions are imposed using ghost cells that are appended to the domain to allow for finite volume calculations at boundaries. Because the plasma is assumed to be spatially infinite, periodic boundary conditions are applied in the $\hat{x}$ direction. Solid-wall boundary conditions are applied at $\left|v_{x}\right|,\left|v_{y}\right|=v_{\max }$ by: setting the value of the distribution function to zero in the velocity-boundary ghost cells i.e. enforcing $f=0$ for $|v|>v_{\max }$; and setting the values of fluxes at velocity boundaries to zero. The latter stipulation ensures that the integral of the distribution function over the entire phase space domain is conserved. The solid-wall boundary condition is valid provided that $f\left(v_{\max }\right)$ is sufficiently close to zero.

\subsection{Fourth-order finite volume discretization}

The non-dimensional Vlasov-Poisson system is solved using an explicit finite volume method with fourth-order accuracy in time and space. Fourth-order interpolations are used for spatial accuracy, and fourth-order Runge-Kutta is used to advance the cell-average distribution function in time.

The probability distribution function is initialized by a fourth-order cell-average of the analytic $\left.f\left(x, v_{x}, v_{y}\right)\right|_{t=0}$, such that the cell-average value is computed as the sum of the cell-center value and second derivative corrections:

$$
\begin{aligned}
\langle f\rangle_{i, j, k}= & f_{i, j, k}+\frac{h_{x}^{2}}{24}\left(\frac{f_{i+1, j, k}-2 f_{i, j, k}+f_{i-1, j, k}}{h_{x}^{2}}\right)+\frac{h_{v_{x}}^{2}}{24}\left(\frac{f_{i, j+1, k}-2 f_{i, j, k}+f_{i, j-1, k}}{h_{v_{x}}^{2}}\right) \\
& +\frac{h_{v_{y}}^{2}}{24}\left(\frac{f_{i, j, k+1}-2 f_{i, j, k+1}+f_{i, j, k-1}}{h_{v_{y}}^{2}}\right)
\end{aligned}
$$

where $\langle\cdot\rangle$ denotes the cell-average value, subscripts $i, j, k$ denote discretized domain cell indices, and $h_{x}, h_{v_{x}}$ and $h_{v_{y}}$ are the grid spacings in the $\hat{x}, \hat{v}_{x}$, and $\hat{v}_{y}$ directions, respectively.

For each time step, the algorithm for advancing the cell-average distribution function is:

1. The Poisson equation is solved for the cell-average potential $\langle\phi\rangle_{i}$ using a 4th-order finite volume stencil:

$$
-\left(-\frac{1}{12}\langle\phi\rangle_{i+2}+\frac{4}{3}\langle\phi\rangle_{i+1}-\frac{5}{2}\langle\phi\rangle_{i}+\frac{4}{3}\langle\phi\rangle_{i-1}-\frac{1}{12}\langle\phi\rangle_{i-2}\right)=1-\sum_{j} \sum_{k}\langle f\rangle_{i, j, k} h_{v_{x}} h_{v_{y}},
$$


with the constraint $\sum_{i}\langle\phi\rangle_{i}=0$, which is used as a means to ensure that the net electric field in the domain is zero.

2. The cell-average electric field $\langle E\rangle_{i}$ is computed using the cell-average potential:

$$
\langle E\rangle_{i}=-\frac{8}{12 h_{x}}\left(\langle\phi\rangle_{i+1}-\langle\phi\rangle_{i-1}\right)+\frac{1}{12 h_{x}}\left(\langle\phi\rangle_{i+2}-\langle\phi\rangle_{i-2}\right) .
$$

3. The cell-average values of the distribution function are interpolated to cell-faces, using a one-point upstream-centered stencil:

$$
\begin{aligned}
& f_{i+\frac{1}{2}, j, k}^{x \text {-face }}= \begin{cases}\frac{2}{60} f_{i-2, j, k}-\frac{13}{60} f_{i-1, j, k}+\frac{47}{60} f_{i, j, k}+\frac{27}{60} f_{i+1, j, k}-\frac{3}{60} f_{i+2, j, k} & \text { for } v_{x}>0 \\
-\frac{3}{60} f_{i-1, j, k}+\frac{27}{60} f_{i, j, k}+\frac{47}{60} f_{i+1, j, k}-\frac{13}{60} f_{i+2, j, k}+\frac{2}{60} f_{i+3, j, k} & \text { for } v_{x} \leq 0\end{cases} \\
& f_{i, j+\frac{1}{2}, k}^{v_{x} \text {-face }}= \begin{cases}\frac{2}{60} f_{i, j-2, k}-\frac{13}{60} f_{i, j-1, k}+\frac{47}{60} f_{i, j, k}+\frac{27}{60} f_{i, j+1, k}-\frac{3}{60} f_{i, j+2, k} & \text { for } Z_{e}\left(E_{x}+v_{y} \frac{\omega_{c}}{\omega_{p}}\right)>0 \\
-\frac{3}{60} f_{i, j-1, k}+\frac{27}{60} f_{i, j, k}+\frac{47}{60} f_{i, j+1, k}-\frac{13}{60} f_{i, j+2, k}+\frac{2}{60} f_{i, j+3, k} & \text { for } Z_{e}\left(E_{x}+v_{y} \frac{\omega_{c}}{\omega_{p}}\right) \leq 0\end{cases} \\
& f_{i, j, k+\frac{1}{2}}^{v_{y} \text {-face }}= \begin{cases}\frac{2}{60} f_{i, j, k-2}-\frac{13}{60} f_{i, j, k-1}+\frac{47}{60} f_{i, j, k}+\frac{27}{60} f_{i, j, k+1}-\frac{3}{60} f_{i, j, k+2} & \text { for } v_{x} \frac{\omega_{c}}{\omega_{p}}>0 \\
-\frac{3}{60} f_{i, j, k-1}+\frac{27}{60} f_{i, j, k}+\frac{47}{60} f_{i, j, k+1}-\frac{13}{60} f_{i, j, k+2}+\frac{2}{60} f_{i, j, k+3} & \text { for } v_{x} \frac{\omega}{c}_{\omega_{p}} \leq 0\end{cases}
\end{aligned}
$$

4. Flux terms on the righthand side of Eq. (15) are evaluated using cell-face values of $E, v_{x}, v_{y}, f$, and $B_{z}$ and associated fourth-order corrections [47]:

$$
\begin{aligned}
F_{i+\frac{1}{2}, j, k}^{x \text {-face }}= & f_{i+\frac{1}{2}, j, k}^{x \text {-face }}\left\langle v_{x}\right\rangle_{j}+\frac{h_{v_{x}}}{24}\left(f_{i+\frac{1}{2}, j+1, k}^{x \text {-face }}-f_{i+\frac{1}{2}, j-1, k}^{x \text {-face }}\right) \\
F_{i, j+\frac{1}{2}, k}^{v_{x} \text {-face }}= & f_{i, j+\frac{1}{2}, k}^{v_{x} \text {-face }}\langle E\rangle_{i}+\frac{1}{48}\left(f_{i+1, j+\frac{1}{2}, k}^{v_{x} \text {-face }}-f_{i-1, j+\frac{1}{2}, k}^{v_{x} \text {-face }}\right)\left(\langle E\rangle_{i+1}-\langle E\rangle_{i-1}\right) \\
& +\frac{\omega_{c}}{\omega_{p}}\left[f_{i, j+\frac{1}{2}, k}^{v_{x} \text {-face }}\left\langle v_{y}\right\rangle_{k}+\frac{h_{v_{y}}}{24}\left(f_{i, j+\frac{1}{2}, k+1}^{v_{x} \text {-face }}-f_{i, j+\frac{1}{2}, k-1}^{v_{x} \text {-face }}\right)\right] \\
F_{i, j, k+\frac{1}{2}}^{v_{y} \text {-face }}= & \frac{\omega_{c}}{\omega_{p}}\left[f_{i, j, k+\frac{1}{2}}^{v_{y} \text {-face }}\left\langle v_{x}\right\rangle_{j}+\frac{h_{v_{x}}}{24}\left(f_{i, j+1, k+\frac{1}{2}}^{v_{y} \text {-face }}-f_{i, j-1, k+\frac{1}{2}}^{v_{y} \text {-face }}\right)\right] .
\end{aligned}
$$

Note that $x, v_{x}$, and $v_{y}$ are independent coordinates, electric field depends only on position, and the magnetic field is constant.

5. Eq. (15) is expressed in terms of a time derivative and divergence of fluxes:

$$
\frac{\partial}{\partial t}\langle f\rangle_{i, j, k}=-\frac{1}{h_{x}}\left(F_{i+\frac{1}{2}, j, k}^{x \text {-face }}-F_{i-\frac{1}{2}, j, k}^{x \text {-face }}\right)+\frac{1}{h_{v_{x}}}\left(F_{i, j+\frac{1}{2}, k}^{v_{x} \text {-face }}-F_{i, j-\frac{1}{2}, k}^{v_{x} \text {-face }}\right)-\frac{1}{h_{v_{y}}}\left(F_{i, j, k+\frac{1}{2}}^{v_{y} \text {-face }}-F_{i, j, k-\frac{1}{2}}^{v_{y} \text {-face }}\right) .
$$

6. $\langle f\rangle_{i, j, k}$ is advanced in time using a fourth-order explicit Runge-Kutta method.

The one-point upstream-centered stencil used to interpolate $\langle f\rangle$ to cell faces is an upwindbiased method [48] that takes into account the direction of hyperbolic advection. Thus upwind-biasing provides dissipation to damp out numerical errors with wavelengths $2 h_{x}, 2 h_{v_{x}}$, and $2 h_{v_{y}}$, which are inherent to high-order centered-difference stencils.

For explicit methods the time step is limited by the CFL condition, which depends on grid spacing in each direction $\left(h_{x}, h_{v_{x}}, h_{v_{y}}\right)$ and the fastest advection speed in the system. In this case, the advection speeds that control the distribution function evolution in the $\hat{x}, \hat{v}_{x}$, and $\hat{v}_{y}$ directions are: $\left|v_{x}\right|,\left|E_{x}+v_{y} \frac{\omega_{p}}{\omega_{c}}\right|$, and $\left|v_{x} \frac{\omega_{p}}{\omega_{c}}\right|$, respectively. In phase space calculations, 
velocity is a coordinate and therefore the extent of the domain in the velocity direction imposes a limit on the time step. The limit on the time step size is

$$
\Delta t \leq \sigma \cdot \min \left\{\frac{h_{x}}{v_{\max }}, \frac{h_{v_{x}}}{|E|_{\max }+\frac{\omega_{c}}{\omega_{p}} v_{\max }}, \frac{h_{v_{y}}}{\frac{\omega_{c}}{\omega_{p}} v_{\max }}\right\},
$$

where $|E|_{\max }$ is the maximum of the absolute value of the electric field at any given time and $\sigma$ is the CFL number. The described high-order upwind-biased spatial discretization sets a constraint on the CFL, such that $\sigma$ must be less than $\approx 0.6$ to ensure that the method is numerically stable.

\subsection{Initial condition and its influence on the dominant mode}

The initial condition for the Dory-Guest-Harris instability involves an equilibrium velocity distribution function specified in Eq. (12), and a position-dependent sinusoidal perturbation which constitutes the electrostatic wave. It is important to note that the dependence of the perturbation on $v_{x}$ and $v_{y}$ is arbitrary; however, this dependence dictates how strongly the dominant, fastest-growing mode is excited. This is related to the fact that $\tilde{\omega}_{i}>0$ solutions to the dispersion relation identify the growth rate of the fastest growing mode, whereas other modes will exhibit smaller growth rates. A perturbation with an arbitrary velocity dependence leads to a growth of many different modes, only one of which, over a long period of time, emerges as the dominant mode with the largest growth rate.

In order to determine the velocity dependence of a perturbation that more strongly excites the fastest-growing mode, a perturbation that is symmetric in velocity is first investigated, such that the initial condition is

$$
\begin{aligned}
\left.f\left(x, v_{x}, v_{y}\right)\right|_{t=0} & =\frac{1}{\pi \alpha_{\perp}^{2} j !}\left(\frac{v_{x}^{2}+v_{y}^{2}}{\alpha_{\perp}^{2}}\right)^{j} \exp \left(-\frac{v_{x}^{2}+v_{y}^{2}}{\alpha_{\perp}^{2}}\right)\left(1+\epsilon \sin \left(\frac{\tilde{k} \Omega_{c}}{v_{\perp 0}} x\right)\right) \\
x & \in[0, L] \\
v_{x} & \in\left[-v_{\max }, v_{\max }\right] \\
v_{y} & \in\left[-v_{\max }, v_{\max }\right] \\
\tilde{k} & =\frac{2 \pi}{L} \frac{v_{\perp 0}}{\Omega_{c}}
\end{aligned}
$$

where $\epsilon \ll 1$ is the amplitude of the perturbation. This distribution, with $\tilde{k}$ and $\omega_{p} / \omega_{c}$ for which the dispersion relation predicts a non-zero imaginary component of frequency, is evolved for a time period of several hundred plasma frequencies. Isosurfaces of the peak values of $f\left(x, v_{x}, v_{y}\right)$ at the final time gives an indication of the dominant mode topology. Informed by this qualitative analysis, an initial condition with a perturbation of the following 


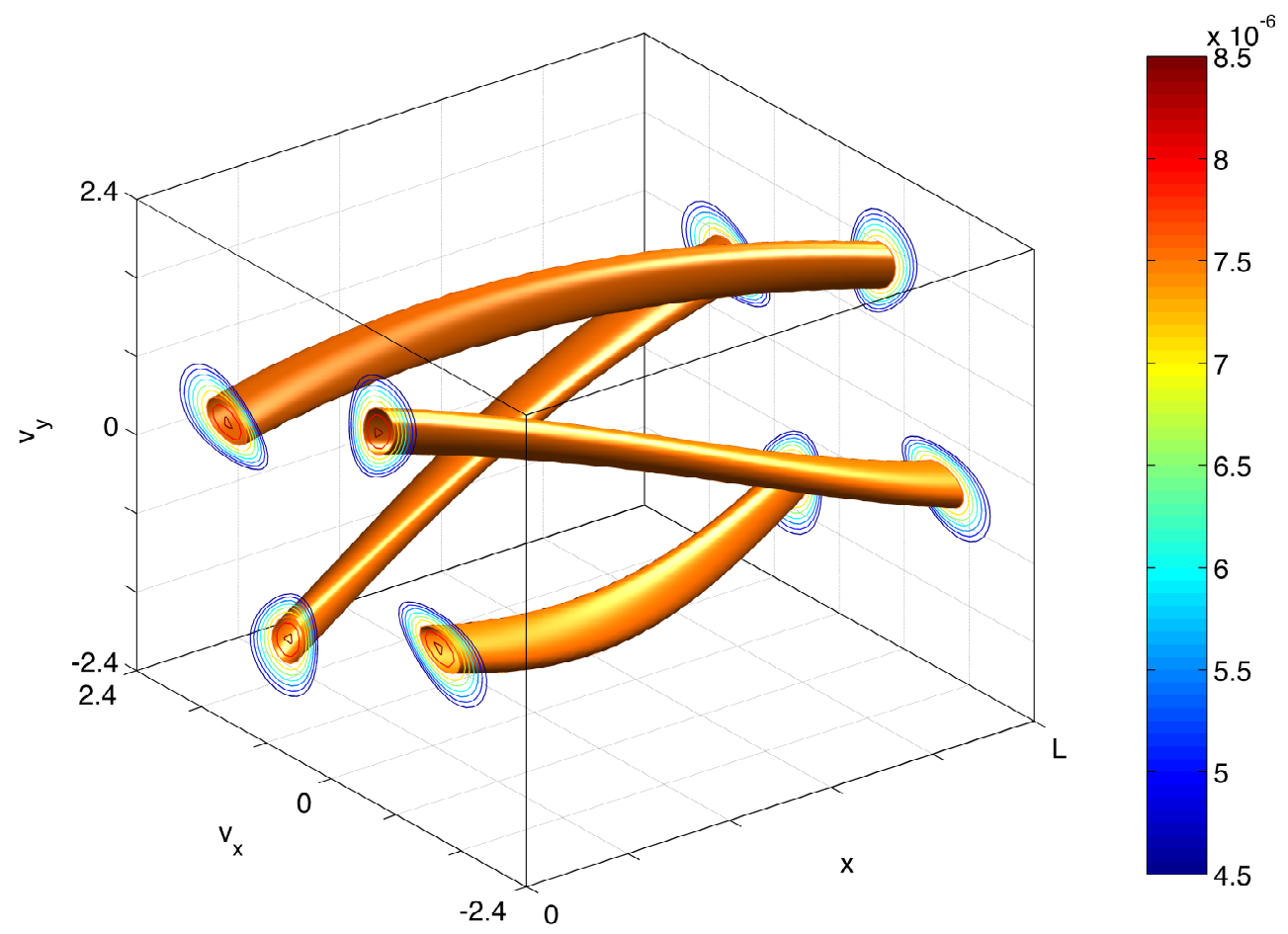

Figure 6: Isosurface $\left(f-f_{0}\right)=7.5 \times 10^{-6}$ in phase space at initial time, depicting the helical structure of the initial perturbation that strongly excites the dominant mode. Contours at $x=0$ and $x=L$ show that the perturbation is periodic along the $\hat{x}$ direction.

form is found to most effectively excite the dominant mode:

$$
\begin{aligned}
\left.f\left(x, v_{x}, v_{y}\right)\right|_{t=0} & =\frac{1}{\pi \alpha_{\perp}^{2} j !}\left(\frac{v_{x}^{2}+v_{y}^{2}}{\alpha_{\perp}^{2}}\right)^{j} \exp \left(-\frac{v_{x}^{2}+v_{y}^{2}}{\alpha_{\perp}^{2}}\right)\left(1+\epsilon \sin \left(4 \theta-\frac{\tilde{k} \Omega_{c}}{v_{\perp 0}} x\right)\right) \\
\theta & =\arctan \left(\frac{v_{y}}{v_{x}}\right) .
\end{aligned}
$$

This perturbation is peaked along a four-stranded helix in phase space coordinates $\left(x, v_{x}, v_{y}\right)$, with a position-dependent sinusoidal wave with wavelength $L$ and a velocity-dependent component that excites the fastest growing mode for cases of $\tilde{\omega}_{i}>0$. See Fig. 6 for a depiction of the peaks of this perturbation.

For all simulation results presented here, the perturbation amplitude is chosen to be $\epsilon=1 \times 10^{-4}$ and parameter $\alpha_{\perp}$ is selected to have a value of $(2 / j)^{1 / 2}$ so that $v_{\perp 0}=\sqrt{2}$. The perturbation amplitude determines how quickly the instability saturates, and thus for smaller $\epsilon$ the linear phase of the instability lasts longer. In general $\alpha_{\perp}$ and $j$ should be selected subject to constraints associated with resolution limits (due to computational cost) and the CFL condition.

Another aspect of the initial condition that deserves particular attention is the normalization. The integral of Eq. (28) and Eq. (29) over the infinite velocity domain is one, 
which ensures that the system being modeled is globally charge neutral. However, for computational cost purposes associated with resolution and the CFL stability limit on the time step size (see Eq. (27)), the velocity domain is truncated such that in simulations $v_{x}, v_{y} \in\left[-v_{\max }, v_{\max }\right]$. In the simulations discussed in this paper, $v_{\max }=4$. Using a higher $v_{\max }$ would introduce a prohibitive computational expense by (a) further limiting the time step size required for numerical stability and (b) requiring more grid cells to properly resolve the distribution function. Thus the integral over the truncated domain, depending on the choice of $v_{\max }$ and the distribution function parameters, may not be normalized to numerical precision.

To address the effects of a truncated velocity domain and to ensure charge neutrality in the system, the initial condition is scaled by a constant factor $\mathcal{A}$ such that the following expression is satisfied to $10^{-15}$ precision:

$$
\mathcal{A} \int_{-v_{\max }}^{v_{\max }} \int_{-v_{\max }}^{v_{\max }} \frac{1}{\pi \alpha_{\perp}^{2} j !}\left(\frac{v_{x}^{2}+v_{y}^{2}}{\alpha_{\perp}^{2}}\right)^{j} \exp \left(-\frac{v_{x}^{2}+v_{y}^{2}}{\alpha_{\perp}^{2}}\right) d v_{x} d v_{y}=1,
$$

where the value of $\mathcal{A}$ depends on the value of $v_{\max }, j$, and $\alpha_{\perp}$. Ideally $v_{\max }$ would be chosen to be sufficiently high that the scale factor $\mathcal{A}$ would not be necessary, i.e. the scale factor would equal one to numerical precision. For the parameters of interest with $j=\{2,4,6\}$, the scale factor value is one to within a precision of $10^{-6}, 10^{-10}$, and $10^{-15}$, respectively.

\section{Simulation of the Dory-Guest-Harris instability}

\subsection{Quantifying instability growth rate from electric field energy evolution}

In order to compare simulation results to the theoretical predictions from the dispersion relations for the Dory-Guest-Harris probability distribution function, potential energy is tracked as a function of time. The nondimensional electrostatic potential energy, i.e. the electric field energy $U_{E}$, is defined analytically as

$$
U_{E}=\frac{1}{2} \int_{0}^{L} E^{2}(x) d x
$$

and in fourth-order accurate discrete form as

$$
U_{E}=\frac{1}{2} \sum_{i}\langle E\rangle_{i}^{2}+\frac{h_{x}^{2}}{12}\left(\frac{\langle E\rangle_{i+1}-\langle E\rangle_{i-1}}{2 h_{x}}\right)^{2} .
$$

In simulations the Dory-Guest-Harris instability is evidenced by net exponential growth of electrostatic potential energy as a function of time. According to the dispersion relation in Eq. (10), this occurs for waves whose normalized wave numbers yield a positive imaginary frequency, i.e. $\tilde{\omega}_{i}(\tilde{k})>0$. The growth rate of electric field energy in a simulation is determined by fitting an exponential to $U_{E}(t)$, or equivalently finding the slope $S$ of the line that fits $\log \left(U_{E}\right)$ versus time during the linear stage of evolution. In the non-dimensional system of equations being solved, time $t$ is also non-dimensional and is defined to be the product 
of physical time and the electron plasma frequency. Note that it is the growth rate of the electric field, not electric field energy, that is compared against the value of $\tilde{\omega}_{i}^{\text {th }}$ predicted by the linear theory dispersion relation. The growth rate of the electric field is a factor of two smaller than the growth rate of the electric field energy. From the numerical simulations, the electric field growth rate $\tilde{\omega}_{i}^{\text {num }}$ is calculated as

$$
\tilde{\omega}_{i}^{\text {num }}=\frac{1}{2} \frac{\omega_{p}}{\omega_{c}} S
$$

where the $\omega_{p} / \omega_{c}$ factor is necessary for proper normalization. When $\tilde{\omega}_{i}>0, \tilde{\omega}_{r}=0$, pure exponential growth of the electric field is observed, until the instability enters the nonlinear regime and saturates. When $\tilde{\omega}_{i}>0, \tilde{\omega}_{r}>0$, the electric field energy oscillates at a frequency $2 \tilde{\omega}_{r}$ and grows at a rate of $2 \tilde{\omega}_{i}$, which means the electrostatic wave propagates and amplifies until it saturates. From the simulations, the oscillation frequency $\tilde{\omega}_{r}^{\text {num }}$ of the electric field is determined from $U_{E}(t)$

$$
\tilde{\omega}_{r}^{\text {num }}=\frac{\pi}{T} \frac{\omega_{p}}{\omega_{c}}
$$

where $T$ is the period of $U_{E}(t)$ oscillation in units of normalized time, and the ratio of plasma frequency to cyclotron frequency is necessary to be consistent with Eq. (13).

When potential energy oscillates without net growth or decay $\left(\tilde{\omega}_{i}=0\right)$, the electrostatic wave is stable and propagates unchanged through the infinite plasma. In this case $\tilde{\omega}_{r}^{\text {num }}$ is difficult to determine from the simulation, since many different modes are excited by the initial perturbation, none of which grow in time. Exciting a single mode would require solving for the eigenfunction associated with the linearized Vlasov-Poisson system. Such analysis is beyond the scope of this paper and is not necessary to determine stability properties and characterize the behavior of the instability. Cases of stability are therefore verified not by comparing $\tilde{\omega}_{r}^{\text {num }}$ to the theoretically predicted $\tilde{\omega}_{r}^{\text {th }}$, but by verifying that there is no net growth of potential energy as a function of time. Figure 7 shows the electric field energy evolution for the three different cases discussed.

\subsection{Discussion of fourth-order simulation results}

Cases of stability allow for a qualitative comparison between simulation results and theoretical predictions. The Maxwellian equilibrium distribution function is verified to be stable for different values of $\alpha_{\perp}$ and $\tilde{k}$. This result is consistent with those presented in Ref. [31]. Other parameter values for which linear theory predicts stability are listed in Table 1 and are verified to be stable in simulations. Note that these parameters correspond to cases for which frequency is real-valued - see Fig. 3, Fig. 4, and Fig. 5 for the dispersion diagrams. For each set of stable parameters, the electric field energy exhibits no net change in magnitude. Figure 7 shows the electric field energy evolution for the stable case of $j=2$, $\tilde{k}=2.12$, and $\omega_{p} / \omega_{c}=10$ as compared to unstable cases, for which field energy grows by many orders of magnitude.

For parameter values that result in growth of the instability, simulation results can be quantitatively compared against theoretical predictions. See Table 2 for a comparison of 


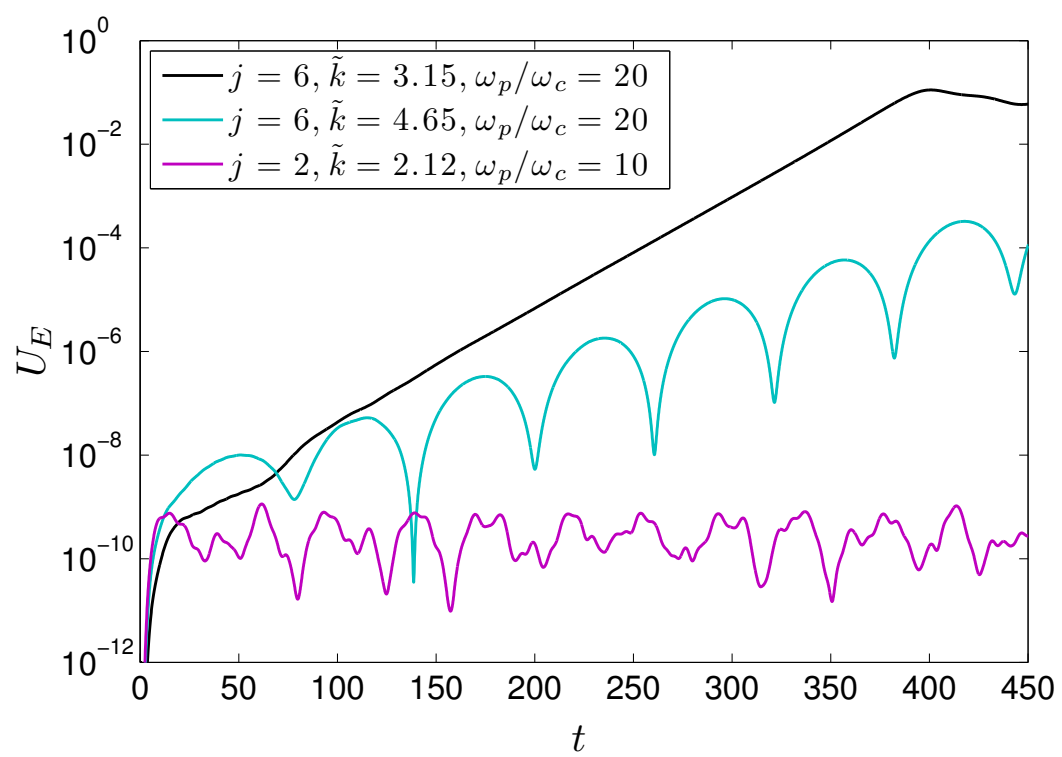

Figure 7: Comparison of electric field energy evolution for cases in which perturbation: amplifies only (black), amplifies and propagates (cyan), and propagates only (purple). Time is normalized by the electron plasma frequency.

Table 1: Sets of parameters $j, \omega_{p} / \omega_{c}$, and $\tilde{k}$ for which simulations show stability of the Dory-Guest-Harris distribution in response to perturbation.

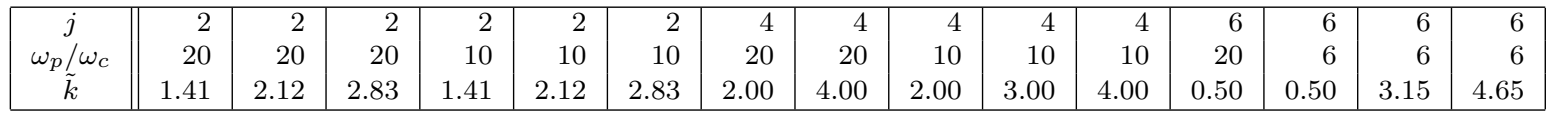


Table 2: Converged values of frequencies from simulations compared to theoretical values predicted by the dispersion relation for parameters that result in instability. Error is defined as the relative difference between the theoretical value and the converged value from fourthorder simulations, e.g. $\left|\tilde{\omega}_{i}^{\text {th }}-\tilde{\omega}_{i}^{\text {num }}\right| / \tilde{\omega}_{i}$.

\begin{tabular}{rrr|rrr|rrr}
$j$ & $\omega_{p} / \omega_{c}$ & $\tilde{k}$ & $\tilde{\omega}_{i}^{\text {th }}$ & $\tilde{\omega}_{i}^{\text {num }}$ & Error & $\tilde{\omega}_{r}^{\text {th }}$ & $\tilde{\omega}_{r}^{\text {num }}$ & Error \\
\hline \hline 4 & 20 & 3.00 & 0.2938 & 0.3003 & $2.2 \%$ & - & - & - \\
6 & 20 & 3.15 & 0.4912 & 0.4958 & $0.9 \%$ & - & - & - \\
6 & 20 & 4.65 & 0.2899 & 0.2903 & $0.1 \%$ & 1.0361 & 1.0310 & $0.5 \%$
\end{tabular}

frequency values obtained from simulation against dispersion relation predictions. For the case of pure growth with $j=4, \tilde{k}=3.00$, and $\omega_{p} / \omega_{c}=20$ the simulation-derived growth rate is 0.3003 , which is $2.2 \%$ larger than the theoretical value. Simulated electric field energy evolution as a function of time for $j=6$ is shown in Fig. 8(a) for the case of pure growth, with $\tilde{k}=3.15$ and $\omega_{p} / \omega_{c}=20$. For these parameters, the electrostatic wave does not propagate and its amplitude grows exponentially in time at a rate of $\tilde{\omega}_{i}^{\text {num }}=0.4958$. This growth rate value is within one percent of the theoretical value $\tilde{\omega}_{i}^{\text {th }}=0.4912$ predicted by the dispersion relation. Figure 9(a) shows electric field energy evolution for the case of growth with propagation, with $j=6, \tilde{k}=4.65$ and $\omega_{p} / \omega_{c}=20$. For this set of parameters the perturbation grows at a rate of $\tilde{\omega}_{i}^{\text {num }}=0.2903$ and oscillates at a frequency of $\tilde{\omega}_{r}^{\text {num }}=1.0310$. These values are within one percent of the theoretical predictions (see Table 2).

All values of simulation-derived frequencies presented in this paper are converged from simulation outputs for three different grid resolutions. Thus for a given set of simulation parameters, the fourth-order electric field energy outputs are generated using grids of size $N_{x} \times N_{v_{x}} \times N_{v_{y}}: 32 \times 64 \times 64,40 \times 80 \times 80$, and $48 \times 96 \times 96$. The number of cells $N_{v_{x}}$ in the $\hat{v}_{x}$ direction and $N_{v_{y}}$ in the $\hat{v}_{y}$ directions are equal such that the velocity grid spacings are uniform, $h_{v_{x}}=h_{v_{y}}=h_{v}$. As the grid is refined, $h_{v} \rightarrow 0$ and $\tilde{\omega}^{\text {num }}$ approaches a limiting value. The value $h_{v}^{c}$ is the lowest resolution grid cell spacing, corresponding to the $32 \times 64 \times 64$ grid. Converged fourth-order simulation results for cases of instability growth are presented in Figs. 8(b), 9(b), and 9(c).

Representative convergence study results are shown in Fig. 8(b) for a purely imaginary frequency, and in Figs. 9(b) and 9(c) for a mixed complex frequency. The convergence study enables one to find values of $\tilde{\omega}_{r}^{\text {num }}$ and $\tilde{\omega}_{i}^{\text {num }}$ in the limit of infinite resolution and to verify the accuracy of the numerical method. Since the discrete numerical representation of the Vlasov-Poisson partial differential equation system is an approximation with order accuracy $p$, the error is expected to scale as $\left(h_{v}\right)^{p}$. For the finite volume method described in Sec. 4.2, $p=4$. Thus, simulation-derived frequency values as a function of $\left(h_{v}\right)^{4}$ should be a linear relation such that the $y$-intercept is the converged value of frequency. The data in Figs. 8(b), 9 (b), and 9(c) are well represented by line fits, which verifies that the numerical method is fourth-order accurate.

Figures 8(a) and 9(a) show that the instability evolution enters a nonlinear regime and saturates. Saturation occurs when the electric field energy reaches a value on the order of 


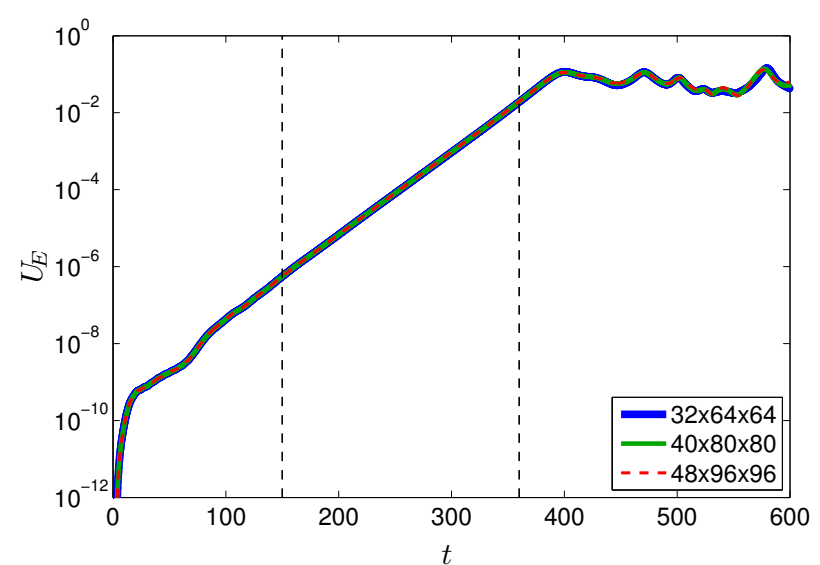

$((\mathrm{a}))$

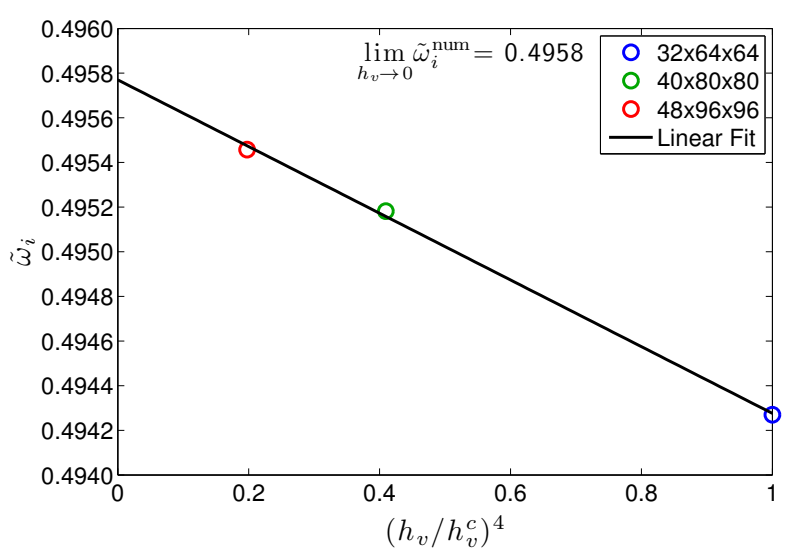

$((\mathrm{b}))$

Figure 8: Fourth-order simulation results with $j=6, \omega_{p} / \omega_{c}=20$, and $\tilde{k}=3.15$. ((a)) Electric field energy grows exponentially in time. Linear phase is observed for time $t \in$ $[150,360]$, denoted by the vertical dashed lines. Time is normalized by the electron plasma frequency. ((b)) As the simulation grid is refined from $32 \times 64 \times 64$ to $48 \times 96 \times 96$, the growth rate converges at fourth-order to a value of 0.4958 .

$10^{-2}$. This saturation threshold is consistent with PIC simulations for a delta function ring initial condition [29]. Figure 10 shows contour slices of the electron probability distribution function in phase space during the nonlinear phase of the evolution for parameters $j=6, \tilde{k}=$ $4.65, \omega_{p} / \omega_{c}=20$. The figure shows that the Dory-Guest-Harris instability is characterized by a complicated 3D structure in phase space with evidence of phase mixing due to the combined effects of shearing and Larmor motion. The probability distribution function, averaged over position $x$, exhibits increased velocity spread over time. For the warm ring initial conditions presented here, this effect is subtle during the linear stage of evolution, but becomes more pronounced during the nonlinear regime. As a point of comparison, deltafunction ring distributions simulated using a PIC method also exhibit increased velocity spread, leading up to and following saturation [29].

\subsection{Order of accuracy considerations}

To assess the effect of numerical method accuracy on the simulation results, a secondorder variant of the fourth-order finite volume method discussed in Sec. 4.2 is implemented. The second-order method uses second-order explicit Runge Kutta time advance and smaller stencils for the spatial finite volume discretization. Thereby it takes less numerical operations and achieves lower computational cost at the expense of spatial and temporal accuracy.

Second-order simulations with $j=6, \tilde{k}=3.15$, and $\omega_{p} / \omega_{c}=20$ exhibit electric field energy evolution that is similar to the fourth-order results presented in Fig. 8(a). The linear phase of the instability visibly depends on the grid resolution, but otherwise exhibits the same features: the linear phase starts at time $t=150$ and saturation occurs when electric field energy reaches $10^{-2}$. Fig. 11 shows the instability growth rates as derived 


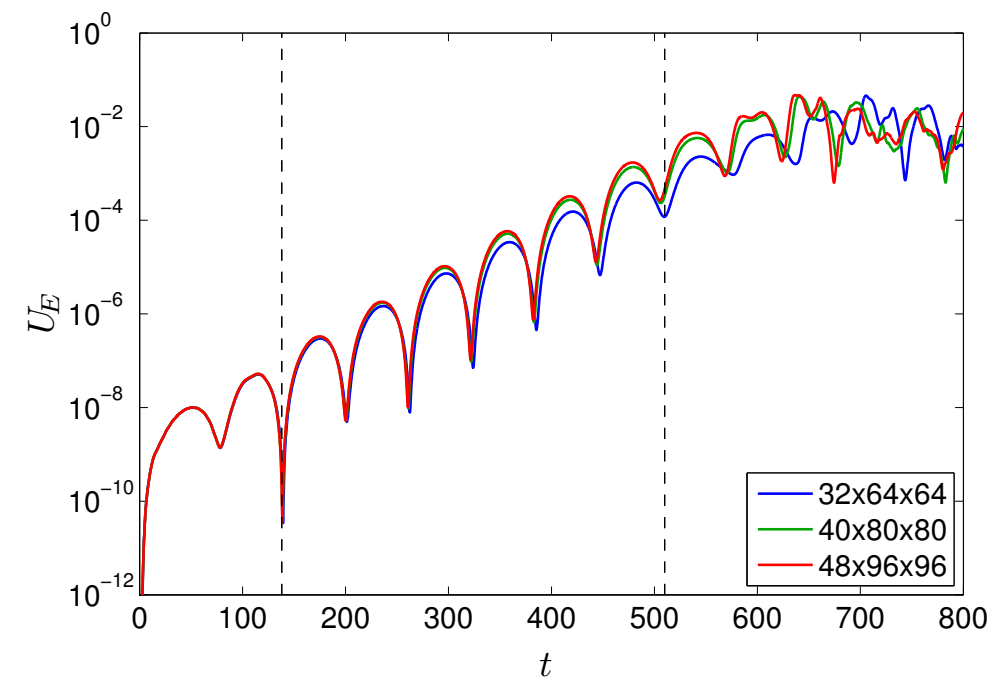

$((\mathrm{a}))$

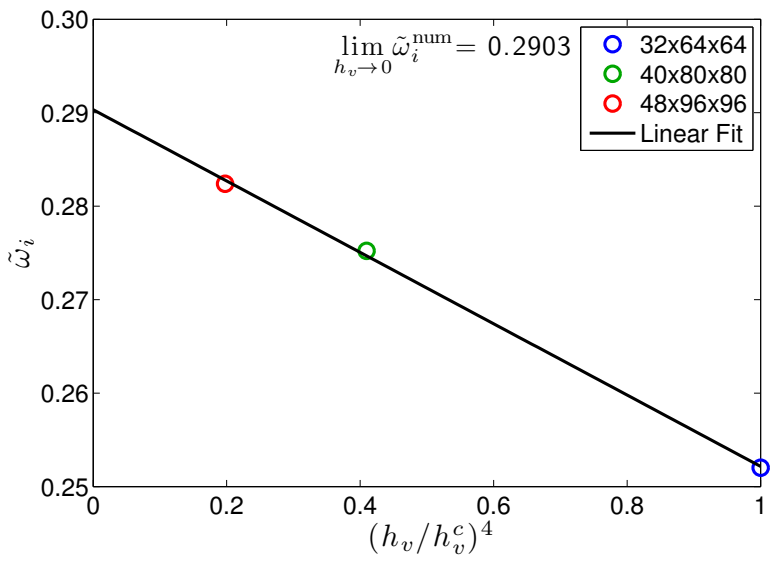

$((\mathrm{b}))$

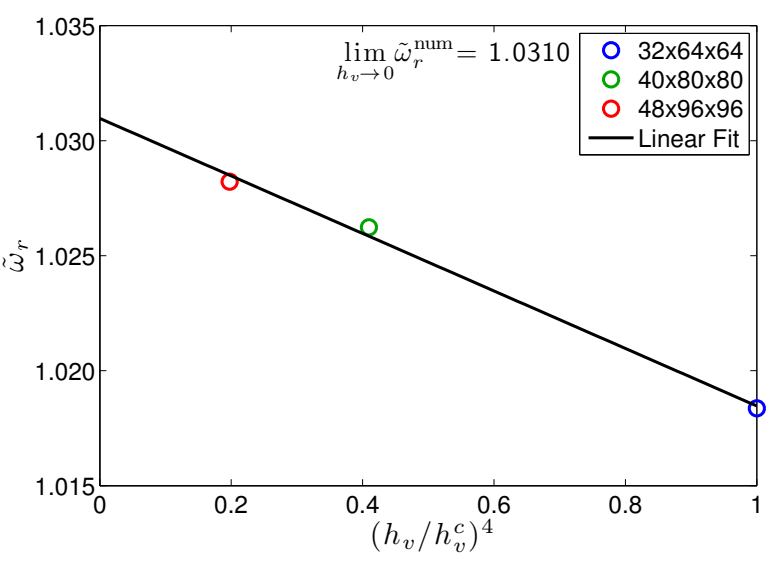

$((\mathrm{c}))$

Figure 9: Fourth-order simulation results with $j=6, \omega_{c} / \omega_{p}=20$, and $\tilde{k}=4.65$ using different grid resolutions.((a)) The Dory-Guest-Harris equilibrium distribution function is unstable and the initial small-amplitude wave grows and propagates in time. Propagation is evidenced by the periodic oscillations in the electric field energy. The linear phase of the instability is observed for $t \in[138,510]$ and is denoted by vertical black dashed lines. Time is normalized by the electron plasma frequency. ((b)) As the simulation grid is refined instability growth rate converges at fourth-order to a value of 0.2903. ((c)) Real frequency converges at fourth-order to a value of 1.0310. 

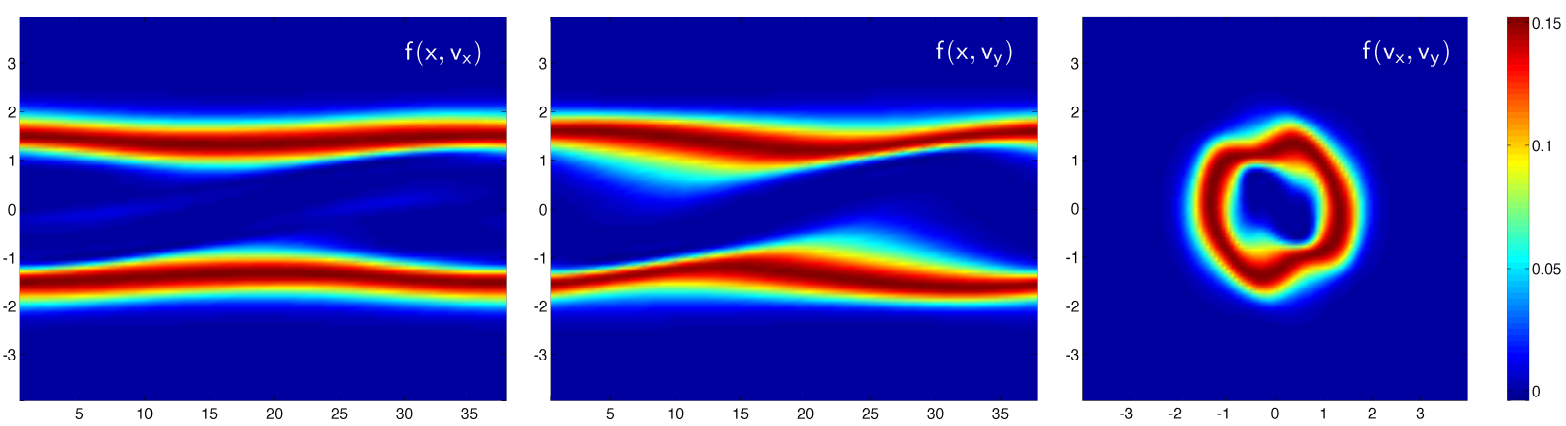

((a)) $t=537.6$
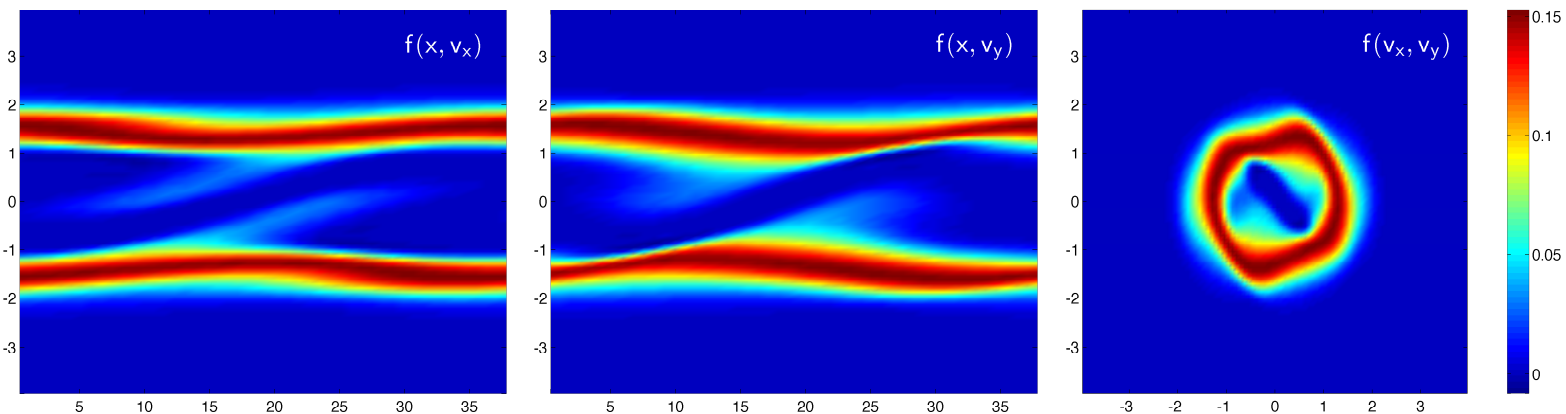

((b)) $t=553.7$
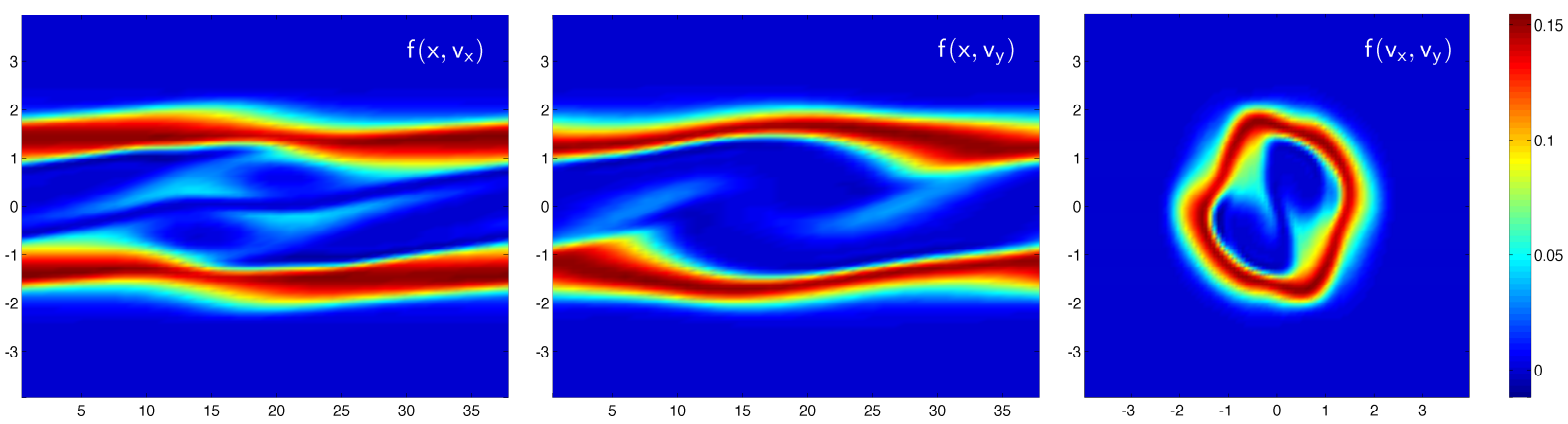

((c)) $t=585.9$
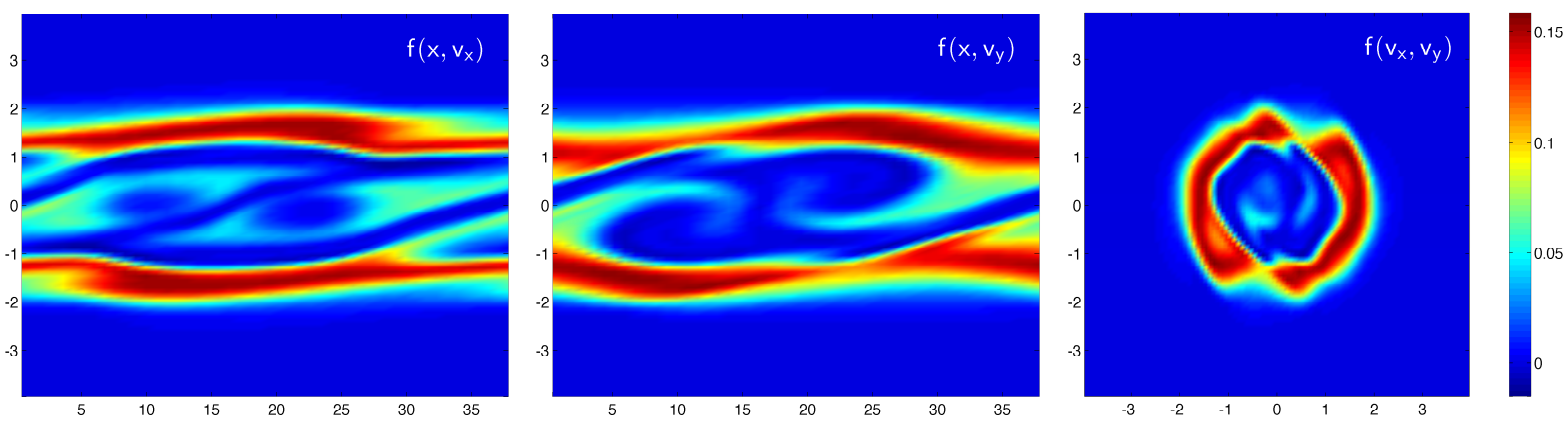

$((\mathrm{d})) t=618.2$

Figure 10: Contours showing the fourth-order accurate nonlinear evolution of the distribution function on a $48 \times 96 \times 96$ grid with $j=6, \tilde{k}=4.65, \omega_{p} / \omega_{c}=20$ with slices at $v_{y}=0, v_{x}=0$, and $x=L / 2$. Negative values of the distribution function are an unphysical numerical artifact. Time is normalized by the electron plasma frequency. 
from the second-order simulations using different grid resolutions. The value of the growth rate is a linear function of $\left(h_{v}\right)^{2}$, verifying that the rate of convergence is second-order. The converged value of the instability growth rate in this case is 0.5413 , which is $10 \%$ off from the theoretically-predicted value shown in Table 2. The large error is due to the lower accuracy of the method and can be improved with higher resolution.

Second-order simulations of instability growth and propagation with $j=6, \tilde{k}=4.65$, and $\omega_{p} / \omega_{c}=20$ are shown in Fig. 12. In this case grid resolutions $32 \times 64 \times 64$ and $40 \times 80 \times 80$ are insufficient and fail to capture the growth and propagation of the initial electric field perturbation. Higher resolution is required in order to reach the asymptotic regime and demonstrate convergence of the second-order method. Even when using a resolution of $64 \times 128 \times 128$, the second-order method is unable to capture the transition between the linear and non-linear phases of instability evolution. Consequently the evolution of the electric field energy starts to exhibit nonlinear behavior long before the instability is expected to saturate. As the grid is refined from $48 \times 96 \times 96$ to $64 \times 128 \times 128$, the imaginary component of frequency converges to a value of 0.3693 which is $27 \%$ off from the theoretically predicted value (see Table 2 ). The converged value of the oscillation frequency, $\tilde{\omega}_{r}^{\text {num }}=1.0445$ is $0.8 \%$ off from the theoretically predicted value. As in the case of pure growth, large error in the growth rate can be mitigated by using higher resolution.

As expected, second-order simulation results are consistent with fourth-order results, but require higher resolution to achieve adequate convergence for the value of the growth rate. Cases in which the instability amplifies and propagates are particularly sensitive to the order of accuracy. Thus low order methods can successfully be benchmarked against the Dory-Guest-Harris instability, provided the resolution is high enough to ensure that a given simulation is in the asymptotic regime.

\subsection{Sources of error}

While converged values of fourth-order simulation-derived frequencies closely agree with theoretical values predicted by the dispersion relation, an assessment of simulation limitations and sources of error is warranted. One source of error in the simulations is the truncation of velocity boundaries. Truncation is necessitated by the CFL condition, which limits time step size based on the value of $v_{\max }$ - a unique feature of phase space calculations (see Eq. (27)). When the velocity domain is truncated, the value of the distribution function at the velocity boundary can be appreciably greater than zero, which affects the validity of the imposed velocity boundary conditions. Note that $j, \alpha_{\perp}$ and the re-normalization of the distribution function after velocity boundary truncation (see Eq. (31)) affect the value of $f\left(v_{\max }\right)$. Since solid wall boundary conditions at $\left|v_{x}\right|,\left|v_{y}\right|=v_{\max }$ are imposed by setting ghost cell values and fluxes to zero, truncation does not affect conservation. Thus, in the finite volume simulation results presented, mass (i.e. the zeroth velocity moment of the distribution function) is conserved to numerical precision. Truncation does, however, introduce an unphysical barrier that can impact the solution. This may explain why the $j=4$ test case, which has a higher value of $f\left(v_{\max }\right)$, exhibits the worst agreement with theoretical predictions. Using a higher value of $v_{\max }$ would resolve discrepancies due to boundary effects 


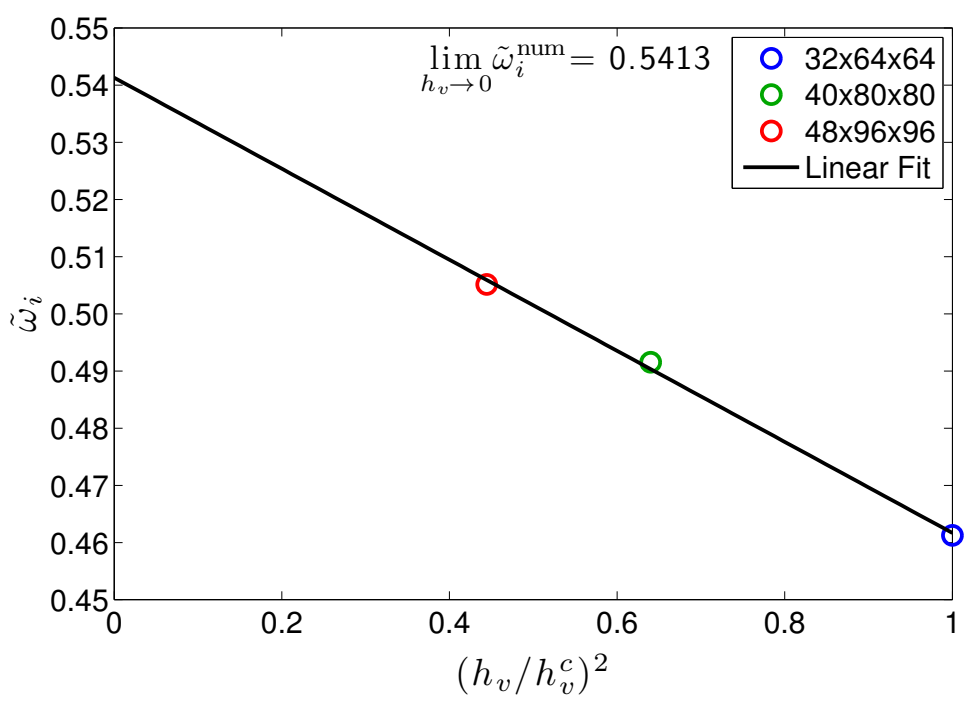

Figure 11: Second-order simulation results for the case of pure growth with $j=6, \omega_{p} / \omega_{c}=$ 20 , and $\tilde{k}=3.15$. As the simulation grid is refined from $32 \times 64 \times 64$ to $48 \times 96 \times 96$, the growth rate converges at second-order to a value of 0.5413 .

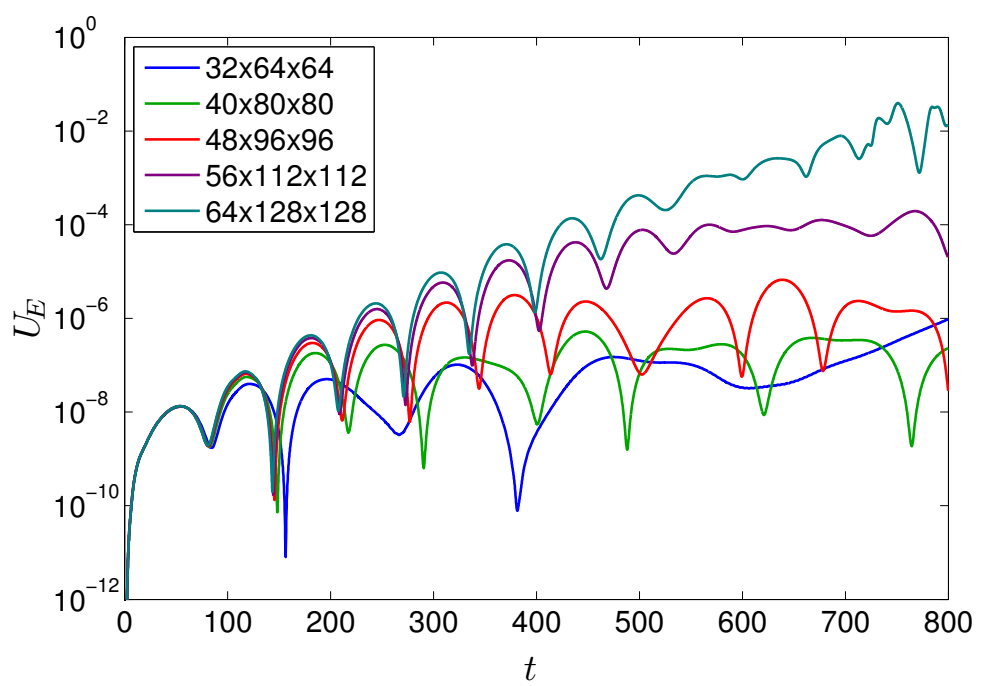

Figure 12: Second-order simulation results with $j=6, \omega_{p} / \omega_{c}=20$, and $\tilde{k}=4.65$. In order to adequately capture the electric field energy growth and oscillation associated with the linear phase of the Dory-Guest-Harris instability, the second-order method requires higher resolution. 
at the expense of increased computational cost. Equilibrium distribution function parameters $j$ and $\alpha_{\perp}$ can also be modified to decrease the value of $f\left(v_{\max }\right)$ without increasing the value of $v_{\max }$, and thereby improve agreement between theoretical and numerical values of frequency. It is important, however, to ensure that the distribution function features are adequately resolved by the simulation grid.

Numerical errors are another source of discrepancy. These cause the probability distribution function to become negative in regions far away from the distribution function peaks (as evidenced in the minimum values of $f$ shown in Fig. 10). These negative values are unphysical and are the result of under-resolved gradients associated with the spatial discretization. Consequently the errors can be diminished by increasing the resolution. Since fine-scale phase space features develop progressively throughout a simulation, increased resolution would also extend the duration over which the instability can be modeled. Positivity preserving methods involving limiters can also be used to mitigate the effects of these numerical errors.

Low resolution also limits the accuracy of the convergence study - both for high-order and low-order numerical methods. Second-order simulations in particular require higher resolution in order to converge to a theoretically predicted solution. The grid resolution is limited by the computational cost of advancing the distribution function in three dimensions. Higher resolution simulations, facilitated by optimization of algorithm performance, would likely improve the accuracy of the convergence fits (see Figs. 8(b), 9(b), and 9(c)), and thereby reduce the error in the converged values of $\tilde{\omega}_{i}^{\text {num }}$ and $\tilde{\omega}_{r}^{\text {num }}$. Even with limited resolution, however, fourth-order simulation results demonstrate good convergence, implying that higher resolution calculations may not yield any additional physical insight.

Finally, it is important to note that a nonlinear equation system and nonlinear discretization are being used to simulate a linear problem. Thus discrepancies between the simulation results and dispersion relation predictions are expected as the assumptions used in the linearization process become invalid. Despite the limitations of the algorithm with regard to resolution, truncation, and numerical error, it is shown to successfully model the Dory-Guest-Harris instability, as validated against linear theory.

\section{Conclusions}

The Dory-Guest-Harris instability is demonstrated to be a well-suited benchmark for continuum kinetic Vlasov-Poisson algorithms in 3D $\left(x, v_{x}, v_{y}\right)$ phase space. It has a closedform linear theory dispersion relation that can be used to identify thresholds for instability, against which continuum method simulations can be quantitatively compared. This is done by initializing a warm ring velocity distribution function with a position-dependent sinusoidal wave perturbation. The velocity spread of the distribution function, the wave number of the perturbation, and the ratio of electron plasma frequency to electron cyclotron frequency determine whether an instability occurs. In cases of instability, the electric field energy grows exponentially in time, and the associated growth rate and oscillation frequency can be deduced and compared against linear theory predictions. 
A fourth-order accurate finite volume continuum Vlasov-Poisson algorithm is successfully benchmarked using the presented analysis of the Dory-Guest-Harris distribution function. In cases of stability, the simulation results exhibit no net change in electric field energy. For parameter values that yield an instability, convergence studies are performed to compute real and imaginary frequency components in the limit of infinite resolution. The same convergence studies are used to verify the order of accuracy of the algorithm. The resulting real and imaginary values of frequency are shown to closely agree with theoretical predictions. Discrepancies between simulation outputs and theory are attributed to a number of factors including computational cost limitations, truncation of velocity boundaries, and numerical error. In spite of these factors, growth rates and oscillation frequencies obtained from fourthorder simulations fall within three percent of the values predicted by the dispersion relation.

Results from second-order accurate finite volume simulations indicate that the DoryGuest-Harris instability can be modeled with low-order numerical methods, provided that the resolution is sufficiently high. Since low order methods are less costly, increasing grid resolution as means of improving the level of agreement between simulation results and theoretical predictions is common practice.

As evidenced by the evolution of the Dory-Guest-Harris distribution function, the instability necessitates simulating three dimensions $\left(x, v_{x}, v_{y}\right)$ of phase space. By rotating the distribution function, the benchmark can be used to test methods in four and even six phase space dimensions. Thus the instability provides a means to go beyond the present scope of 2D $\left(x, v_{x}\right)$ standardized benchmark problems, and provides a robust test for extending continuum kinetic algorithms to more phase space dimensions. The described Dory-GuestHarris benchmark is particularly valuable in that it involves a magnetized plasma in which the magnetic field has an important role in the distribution function evolution. The benchmark facilitates a quantitative means by which to evaluate continuum kinetic algorithms, as they become more developed, more robust, and include more generalized physics.

\section{Acknowledgments}

This research was supported by an award from the Department of Energy Office of Science Graduate Fellowship Program (DOE SCGF), a grant from the Office of Advanced Scientific Computing Research of the US Department of Energy under Contract Number DE-AC02-05CH11231, and a grant from the United States Air Force Office of Scientific Research.

\section{Appendix A. Derivation of the closed-form dispersion relation for $k_{\|}=0$ elec- trostatic waves}

The dispersion relation for $k_{\|}=0$ kinetic electrostatic waves is most often derived and expressed in a form that involves infinite sums [28, 34, 49, 44]. Tataronis et al. [31] present without proof an equivalent closed-form expression, a version of which can also be found in Ref. [30]. The closed-form dispersion relation allows for accurate computation of explicit numerical solutions (i.e. frequency as a function of wavenumber) for arbitrary probability 
distribution functions. In effort to fill in the missing details, a complete derivation of the dispersion relation is presented here, including the derivation of the closed-form expression (see Eqs. (10) and (11)) for a plasma with multiple species $s$.

From Eqs. (8) and (9) it is possible to obtain the analytic form of the dispersion relation by applying the procedure discussed in Ref. [34, pp. 341-352] to the non-dimensionalized system of equations that is used in this paper. Starting with the transformed non-dimensionalized Vlasov equation (see Eq. (8)), let $\alpha=-\omega / \Omega_{c s}$ and $\beta=k_{\perp} v_{\perp} / \Omega_{c s}$. Recall that $\Omega_{c s}=$ $Z_{s} \omega_{c} /\left(M_{s} \omega_{p}\right)$. The result is an ordinary differential equation that can be analytically solved for $\hat{f}_{s 1}$ :

$$
0=-i(\alpha+\beta \cos \theta) \hat{f}_{s 1}-\frac{\omega_{p}}{\omega_{c}} \hat{\mathbf{E}}_{1} \cdot \frac{\partial f_{s 0}}{\partial \mathbf{v}}+\frac{\partial \hat{f}_{s 1}}{\partial \theta}
$$

Solving for $\hat{f}_{s 1}$ using an integrating factor yields

$$
\hat{f}_{s 1}=e^{i(\alpha \theta+\beta \sin \theta)} \int_{\theta_{0}}^{\theta} \frac{\omega_{p}}{\omega_{c}} \hat{\mathbf{E}}_{1} \cdot \frac{\partial f_{s 0}}{\partial \mathbf{v}} e^{-i\left(\alpha \theta^{\prime}+\beta \sin \theta^{\prime}\right)} d \theta^{\prime} .
$$

Substituting Eq. (A.2) into Poisson's equation (see Eq. (9)) and noting that $\mathbf{E}=-\nabla \Phi$ and consequently $\hat{\mathbf{E}}_{1}=-i \mathbf{k} \hat{\Phi}_{1}$ yields

$$
0=1+\sum_{s} \frac{Z_{s} \omega_{p}}{k_{\perp}^{2} \omega_{c}} \int\left[e^{i(\alpha \theta+\beta \sin \theta)} \int_{\theta_{0}}^{\theta} i \mathbf{k} \cdot \frac{\partial f_{s 0}}{\partial \mathbf{v}} e^{-i\left(\alpha \theta^{\prime}+\beta \sin \theta^{\prime}\right)} d \theta^{\prime}\right] d \mathbf{v} .
$$

Recall that $f_{s 0}$ is the equilibrium distribution function and that it is azimuthally symmetric such that $\partial f_{s 0} / \partial \theta=0$. Also note that waves that propagate perpendicular to the magnetic field have $k_{\|}=0$. Consequently the dot product term can be expressed as

$$
i \mathbf{k} \cdot \frac{\partial f_{0}}{\partial \mathbf{v}}=i k_{\perp} \frac{\partial f_{0}}{\partial v_{\perp}} \cos \theta^{\prime}=i k_{\perp} \frac{\partial f_{0}}{\partial v_{\perp}} \frac{e^{i \theta^{\prime}}+e^{-i \theta^{\prime}}}{2} .
$$

Substituting Eq. (A.4) into Eq. (A.3) yields

$$
0=1+\sum_{s} \frac{Z_{s} \omega_{p}}{2 k_{\perp} \omega_{c}} \int\left[e^{i(\alpha \theta+\beta \sin \theta)} i \frac{\partial f_{s 0}}{\partial v_{\perp}} \int_{\theta_{0}}^{\theta}\left(e^{i \theta^{\prime}}+e^{-i \theta^{\prime}}\right) e^{-i\left(\alpha \theta^{\prime}+\beta \sin \theta^{\prime}\right)} d \theta^{\prime}\right] d \mathbf{v} .
$$

The integral with respect to $\theta^{\prime}$ is evaluated with the lower limit $\theta_{0}$ selected so that the antiderivative is zero at $\theta^{\prime}=\theta_{0}$. In order to simplify Eq. (A.5), special properties of the Bessel function of the first kind are invoked. These properties are [50, pp. 7,12]

$$
\begin{aligned}
e^{-i \beta \sin \theta} & =\sum_{n=-\infty}^{\infty} J_{n}(\beta) e^{-i n \theta} \\
J_{n+1}(\beta)+J_{n-1}(\beta) & =\frac{2 n}{\beta} J_{n}(\beta) .
\end{aligned}
$$


The resulting dispersion relation is

$$
0=1-\sum_{s} \frac{Z_{s} \omega_{p}}{k_{\perp} \omega_{c}} \int_{-\infty}^{\infty} \int_{0}^{\infty} \int_{0}^{2 \pi}\left[\frac{1}{\beta} \frac{\partial f_{s 0}}{\partial v_{\perp}} \sum_{m, n=-\infty}^{\infty} n J_{m}(\beta) J_{n}(\beta) \frac{e^{i \theta(m-n)}}{(\alpha+n)}\right] v_{\perp} d \theta d v_{\perp} d v_{\|} .
$$

Note that for the purposes of this paper, all physics of interest is independent of $v_{\|}$. Also note that by the orthogonality properties of Bessel functions and the Fourier basis functions, the terms in the summation Eq. (A.8) are zero for all $m \neq n$. The resulting dispersion relation is

$$
0=1+\sum_{s} \frac{Z_{s}^{2}}{M_{s} k_{\perp}^{2}} \int_{0}^{\infty} \frac{\partial f_{0}}{\partial v_{\perp}} \sum_{n=-\infty}^{\infty} J_{n}^{2}\left(\frac{k_{\perp} v_{\perp}}{\Omega_{c s}}\right) \frac{n}{\frac{\omega}{\Omega_{c s}}-n} 2 \pi d v_{\perp}
$$

This result is consistent with $[28,31]$. Let $w_{s}=\omega / \Omega_{c s}$. It is noted by identity that $J_{n}^{2}=J_{-n}^{2}$ (see Eq. (B.1)) and therefore

$$
\begin{aligned}
\sum_{n=-\infty}^{\infty} \frac{n}{w_{s}-n} J_{n}^{2} & =\sum_{n=-\infty}^{-1} \frac{n}{w_{s}-n} J_{n}^{2}+\sum_{n=1}^{\infty} \frac{n}{w_{s}-n} J_{n}^{2} \\
& =\sum_{n=1}^{\infty}\left[\frac{2 n^{2}}{w_{s}^{2}-n^{2}}\right] J_{n}^{2}
\end{aligned}
$$

Consequently the summation indices in Eq. (A.9) can be changed as follows

$$
0=1+\sum_{s} \frac{Z_{s}^{2}}{M_{s} k_{\perp}^{2}} \sum_{n=1}^{\infty} \frac{2 n^{2}}{w_{s}^{2}-n^{2}} \int_{0}^{\infty} \frac{\partial f_{s 0}}{\partial v_{\perp}} J_{n}^{2}\left(\frac{k_{\perp} v_{\perp}}{\Omega_{c s}}\right) 2 \pi d v_{\perp}
$$

Applying integration by parts and noting that $J_{n}(0)=0$ if $n \neq 0$ and that the equilibrium distribution function has compact support such that $\left.f_{s 0}\right|_{v_{\perp}=\infty}=0$ yields

$$
0=1-\sum_{s} \frac{Z_{s}^{2}}{M_{s} k_{\perp}^{2}} \sum_{n=1}^{\infty} \frac{2 n^{2}}{w_{s}^{2}-n^{2}} \int_{0}^{\infty} f_{s 0} \frac{\partial}{\partial v_{\perp}}\left[J_{n}^{2}\left(\frac{k_{\perp} v_{\perp}}{\Omega_{c s}}\right)\right] 2 \pi d v_{\perp}
$$

It can be readily shown that the following equality is satisfied

$$
\frac{w_{s} \sin \left(w_{s} \pi\right)}{w_{s}^{2}-n^{2}}=\int_{0}^{\pi} \cos (n(\tau+\pi)) \cos \left(w_{s} \tau\right) d \tau
$$

Substituting Eq. (A.14) into Eq. (A.13) yields

$0=1-\sum_{s} \frac{Z_{s}^{2}}{M_{s} k_{\perp}^{2}} \int_{0}^{\infty} f_{s 0} \sum_{n=1}^{\infty}\left[2 n^{2} \int_{0}^{\pi} \frac{\cos (n(\tau+\pi)) \cos \left(w_{s} \tau\right)}{w_{s} \sin \left(w_{s} \pi\right)} d \tau\right] \frac{\partial}{\partial v_{\perp}}\left[J_{n}^{2}\left(\frac{k_{\perp} v_{\perp}}{\Omega_{c s}}\right)\right] 2 \pi d v_{\perp}$. 
The infinite sum in the dispersion relation of Eq. (A.15) can be reduced to an integral representation $[30,31,51]$ using an auxiliary Bessel function identity:

$\int_{0}^{\pi} w_{s} \sin \left(w_{s} \tau\right) \sin \tau J_{0}\left(2 z \cos \frac{\tau}{2}\right) d \tau=-\int_{0}^{\pi} \frac{1}{z} \sum_{n=1}^{\infty} 2 n^{2} \frac{\partial}{\partial z}\left[J_{n}^{2}(z)\right] \cos (n(\tau+\pi)) \cos \left(w_{s} \tau\right) d \tau$

See Appendix B for a complete derivation of this identity. The result is a closed-form integral representation of the dispersion relation that is equivalent to Eq. (A.9):

$$
0=1+\sum_{s} \frac{Z_{s}^{2}}{M_{s} \Omega_{c s}^{2}} \int_{0}^{\infty} f_{s 0} \int_{0}^{\pi} \frac{\sin \left(w_{s} \tau\right)}{\sin \left(w_{s} \pi\right)} \sin (\tau) J_{0}\left(2 \frac{k_{\perp} v_{\perp}}{\Omega_{c s}} \sin \frac{\tau+\pi}{2}\right) d \tau 2 \pi v_{\perp} d v_{\perp},
$$

which is consistent with the expressions presented in Refs. [30, 31].

\section{Appendix B. Auxiliary Bessel function identity}

For integer $n$, the following standard identities apply to Bessel functions of the first kind $[50$, pp. $6,12,102]$

$$
\begin{aligned}
J_{-n}(z) & =(-1)^{n} J_{n}(z) \\
J_{n-1}(z)-J_{n+1}(z) & =2 \frac{\partial}{\partial z}\left[J_{n}(z)\right] \\
J_{0}\left(2 z \sin \frac{\tau}{2}\right) & =\sum_{n=0}^{\infty} \Delta_{n} J_{n}(z)^{2} \cos (n \tau) .
\end{aligned}
$$

where $\Delta_{n}$ is defined as

$$
\Delta_{n}= \begin{cases}1 & \text { if } n=0 \\ 2 & \text { if } n \neq 0\end{cases}
$$

An additional auxiliary Bessel function identity is derived by manipulating Eq. (B.3). References [30] and [51] allude to these types of manipulations.

Starting with Eq. (B.3), the identity can be modified as follows

$$
\begin{aligned}
J_{0}\left(2 z \sin \frac{\tau+\pi}{2}\right) & =\sum_{n=0}^{\infty} \Delta_{n} J_{n}(z)^{2} \cos (n(\tau+\pi)) \\
J_{0}\left(2 z \cos \frac{\tau}{2}\right) & =\sum_{n=0}^{\infty} \Delta_{n} J_{n}(z)^{2} \cos (n(\tau+\pi)) .
\end{aligned}
$$

Taking the derivative of Eq. (B.6) with respect to $\tau$ and using identities in Eq. (B.1) and Eq. (B.2) yields

$$
\sin \frac{\tau}{2} J_{1}\left(2 z \cos \frac{\tau}{2}\right)=-\frac{2}{z} \sum_{n=1}^{\infty} n J_{n}^{2}(z) \sin (n(\tau+\pi))
$$


Taking derivative of Eq. (B.7) with respect to $z$ and using Eq. (B.2) again yields $\cos \frac{\tau}{2} \sin \frac{\tau}{2}\left[J_{0}\left(2 z \cos \frac{\tau}{2}\right)-J_{2}\left(2 z \cos \frac{\tau}{2}\right)\right]=\frac{2}{z^{2}} \sum_{n=1}^{\infty} n J_{n}^{2} \sin (n(\tau+\pi))-\frac{2}{z} \sum_{n=1}^{\infty} n \frac{\partial}{\partial z}\left[J_{n}^{2}(z)\right] \sin (n(\tau+\pi))$.

Using Bessel function identity of Eq. (A.7) and noting that $J_{2}\left(2 z \cos \frac{\tau}{2}\right)=\frac{J_{1}\left(2 z \cos \frac{\tau}{2}\right)}{z \cos \frac{\tau}{2}}-$ $J_{0}\left(2 z \cos \frac{\tau}{2}\right)$, Eq. (B.8) can be expressed as

$2 \cos \frac{\tau}{2} \sin \frac{\tau}{2} J_{0}\left(2 z \cos \frac{\tau}{2}\right)-\frac{\sin \frac{\tau}{2}}{z} J_{1}\left(2 z \cos \frac{\tau}{2}\right)=\frac{2}{z^{2}} \sum_{n=1}^{\infty} n J_{n}^{2} \sin (n(\tau+\pi))-\frac{2}{z} \sum_{n=1}^{\infty} n \frac{\partial}{\partial z}\left[J_{n}^{2}(z)\right] \sin (n(\tau+\pi))$.

Multiplying equation Eq. (B.7) by $z^{-1}$ and substituting into Eq. (B.9) results in the cancelation of two of the terms, thus the manipulated identity is now

$$
\begin{aligned}
2 \cos \frac{\tau}{2} \sin \frac{\tau}{2} J_{0}\left(2 z \cos \frac{\tau}{2}\right) & =-\frac{2}{z} \sum_{n=1}^{\infty} n \frac{\partial}{\partial z}\left[J_{n}^{2}(z)\right] \sin (n(\tau+\pi)) \\
\sin \tau J_{0}\left(2 z \cos \frac{\tau}{2}\right) & =-\frac{2}{z} \sum_{n=1}^{\infty} n \frac{\partial}{\partial z}\left[J_{n}^{2}(z)\right] \sin (n(\tau+\pi)) .
\end{aligned}
$$

Taking the derivative of Eq. (B.11) with respect to $\tau$, multiplying both sides by $\cos \left(w_{s} \tau\right)$, and using the product rule of differentiation yields

$\frac{\partial}{\partial \tau}\left[\sin \tau J_{0}\left(2 z \cos \frac{\tau}{2}\right) \cos \left(w_{s} \tau\right)\right]+w_{s} \sin \left(w_{s} \tau\right) \sin \tau J_{0}\left(2 z \cos \frac{\tau}{2}\right)=-\frac{2}{z} \sum_{n=1}^{\infty} n^{2} \frac{\partial}{\partial z}\left[J_{n}^{2}(z)\right] \cos (n(\tau+\pi)) \cos \left(w_{s} \tau\right)$.

Integrating both sides with respect to $\tau$ on the interval $[0, \pi]$ yields the modified Bessel function identity

$\int_{0}^{\pi} w_{s} \sin \left(w_{s} \tau\right) \sin \tau J_{0}\left(2 z \cos \frac{\tau}{2}\right) d \tau=-\int_{0}^{\pi} \frac{1}{z} \sum_{n=1}^{\infty} 2 n^{2} \frac{\partial}{\partial z}\left[J_{n}^{2}(z)\right] \cos (n(\tau+\pi)) \cos \left(w_{s} \tau\right) d \tau$

which is used in the derivation of the $k_{\|}=0$ dispersion relation.

\section{References}

[1] C. Birdsall, A. Langdon, Plasma physics via computer simulation, McGraw-Hill, New York, NY, 1985.

[2] T. Arber, R. Vann, A critical comparison of Eulerian-grid-based Vlasov solvers, Journal of Computational Physics 180 (1) (2002) 339 - 357. doi:http://dx.doi.org/10.1006/jcph.2002.7098. URL http://www.sciencedirect.com/science/article/pii/S0021999102970981

[3] J. Banks, J. Hittinger, A new class of nonlinear finite-volume methods for Vlasov simulation, Plasma Science, IEEE Transactions on 38 (9) (2010) 2198-2207. doi:http://dx.doi.org/10.1109/TPS.2010.2056937. 
[4] F. Filbet, E. Sonnendrücker, Comparison of Eulerian Vlasov solvers, Computer Physics Communications 150 (3) (2003) 247 - 266. doi:http://dx.doi.org/10.1016/S0010-4655(02)00694-X. URL http://www.sciencedirect.com/science/article/pii/S001046550200694X

[5] W. Guo, J.-M. Qiu, Hybrid semi-Lagrangian finite element-finite difference methods for the Vlasov equation, Journal of Computational Physics 234 (0) (2013) 108 - 132. doi:http://dx.doi.org/10.1016/j.jcp.2012.09.014. URL http://www.sciencedirect.com/science/article/pii/S0021999112005451

[6] J.-M. Qiu, A. Christlieb, A conservative high order semi-lagrangian $\{$ WENO $\}$ method for the vlasov equation, Journal of Computational Physics 229 (4) (2010) 1130 - 1149. doi:http://dx.doi.org/10.1016/j.jcp.2009.10.016.

URL http://www.sciencedirect.com/science/article/pii/S0021999109005610

[7] J. A. Rossmanith, D. C. Seal, A positivity-preserving high-order semi-lagrangian discontinuous galerkin scheme for the vlasov-poisson equations, Journal of Computational Physics 230 (16) (2011) 6203 - 6232. doi:http://dx.doi.org/10.1016/j.jcp.2011.04.018.

URL http://www.sciencedirect.com/science/article/pii/S0021999111002579

[8] A. Suzuki, T. Shigeyama, A conservative scheme for the relativistic Vlasov-Maxwell system, Journal of Computational Physics 229 (5) (2010) 1643 - 1660. doi:http://dx.doi.org/10.1016/j.jcp.2009.11.001. URL http://www.sciencedirect.com/science/article/pii/S0021999109006111

[9] N. Besse, G. Latu, A. Ghizzo, E. Sonnendrücker, P. Bertrand, A wavelet-mra-based adaptive semilagrangian method for the relativistic vlasov-maxwell system, Journal of Computational Physics 227 (16) (2008) 7889 - 7916. doi:http://dx.doi.org/10.1016/j.jcp.2008.04.031.

URL http://www.sciencedirect.com/science/article/pii/S0021999108002672

[10] N. Sircombe, T. Arber, VALIS: A split-conservative scheme for the relativistic 2D Vlasov-Maxwell system, Journal of Computational Physics 228 (13) (2009) 4773 - 4788. doi:http://dx.doi.org/10.1016/j.jcp.2009.03.029.

URL http://www.sciencedirect.com/science/article/pii/S0021999109001636

[11] T. Umeda, J.-i. Miwa, Y. Matsumoto, T. K. M. Nakamura, K. Togano, K. Fukazawa, I. Shinohara, Full electromagnetic Vlasov code simulation of the Kelvin-Helmholtz instability, Physics of Plasmas 17 (5) (2010) -. doi:http://dx.doi.org/10.1063/1.3422547.

URL http://scitation.aip.org/content/aip/journal/pop/17/5/10.1063/1.3422547

[12] J. Candy, R. E. Waltz, Velocity-space resolution, entropy production, and upwind dissipation in Eulerian gyrokinetic simulations, Physics of Plasmas (1994-present) 13 (3) (2006) -. doi:http://dx.doi.org/10.1063/1.2184069.

URL http://scitation.aip.org/content/aip/journal/pop/13/3/10.1063/1.2184069

[13] R. Numata, G. G. Howes, T. Tatsuno, M. Barnes, W. Dorland, AstroGK: Astrophysical gyrokinetics code, Journal of Computational Physics 229 (24) (2010) 9347 - 9372. doi:http://dx.doi.org/10.1016/j.jcp.2010.09.006.

URL http://www.sciencedirect.com/science/article/pii/S0021999110005000

[14] A. Thomas, M. Tzoufras, A. Robinson, R. Kingham, C. Ridgers, M. Sherlock, A. Bell, A review of Vlasov-Fokker-Planck numerical modeling of inertial confinement fusion plasma, Journal of Computational Physics 231 (3) (2012) 1051 - 1079, special Issue: Computational Plasma Physics. doi:http://dx.doi.org/10.1016/j.jcp.2011.09.028.

URL http://www.sciencedirect.com/science/article/pii/S0021999111005717

[15] I. Holod, Z. Lin, Statistical analysis of fluctuations and noise-driven transport in particlein-cell simulations of plasma turbulence, Physics of Plasmas (1994-present) 14 (3) (2007) -. doi:http://dx.doi.org/10.1063/1.2673002.

URL http://scitation.aip.org/content/aip/journal/pop/14/3/10.1063/1.2673002

[16] A. B. Langdon, Kinetic theory for fluctuations and noise in computer simulation of plasma, Physics of Fluids 22 (1) (1979) 163-171. doi:http://dx.doi.org/10.1063/1.862452.

URL http://scitation.aip.org/content/aip/journal/pof1/22/1/10.1063/1.862452

[17] W. Nevins, A. Dimits, G. Hammett, W. Dorland, D. E. Shumaker, Discrete particle noise 
in particle-in-cell simulations of plasma microturbulence, Physics of Plasmas 12 (12) (2005) -. doi:http://dx.doi.org/10.1063/1.2118729.

URL http://scitation.aip.org/content/aip/journal/pop/12/12/10.1063/1.2118729

[18] K. Datta, S. Kamil, S. Williams, L. Oliker, J. Shalf, K. Yelick, Optimization and performance modeling of stencil computations on modern microprocessors, SIAM Review 51 (1) (2009) 129-159. arXiv:http://epubs.siam.org/doi/pdf/10.1137/070693199, doi:10.1137/070693199.

URL http://epubs.siam.org/doi/abs/10.1137/070693199

[19] C. Cheng, G. Knorr, The integration of the Vlasov equation in configuration space, Journal of Computational Physics 22 (3) (1976) 330 - 351. doi:http://dx.doi.org/10.1016/0021-9991(76)90053-X. URL http://www.sciencedirect.com/science/article/pii/002199917690053X

[20] F. Valentini, P. Veltri, A. Mangeney, A numerical scheme for the integration of the Vlasov-Poisson system of equations, in the magnetized case, Journal of Computational Physics 210 (2) (2005) 730 751. doi:http://dx.doi.org/10.1016/j.jcp.2005.05.014.

URL http://www.sciencedirect.com/science/article/pii/S0021999105002597

[21] T. Umeda, Y. Omura, P. Yoon, R. Gaelzer, H. Matsumoto, Harmonic Langmuir waves. iii. Vlasov simulation, Physics of Plasmas 10 (2) (2003) 382-391. doi:http://dx.doi.org/10.1063/1.1537240. URL http://scitation.aip.org/content/aip/journal/pop/10/2/10.1063/1.1537240

[22] A. Ghizzo, P. Bertrand, M. Shoucri, T. Johnston, E. Fualkow, M. Feix, A Vlasov code for the numerical simulation of stimulated Raman scattering, Journal of Computational Physics 90 (2) (1990) 431 - 457. doi:http://dx.doi.org/10.1016/0021-9991(90)90174-Y.

URL http://www.sciencedirect.com/science/article/pii/002199919090174Y

[23] F. Califano, T. Cecchi, C. Chiuderi, Nonlinear kinetic regime of the Weibel instability in an electronion plasma, Physics of Plasmas 9 (2) (2002) 451-457. doi:http://dx.doi.org/10.1063/1.1435001. URL http://scitation.aip.org/content/aip/journal/pop/9/2/10.1063/1.1435001

[24] A. Mangeney, F. Califano, C. Cavazzoni, P. Travnicek, A numerical scheme for the integration of the Vlasov-Maxwell system of equations, Journal of Computational Physics 179 (2) (2002) 495 - 538. doi:http://dx.doi.org/10.1006/jcph.2002.7071. URL http://www.sciencedirect.com/science/article/pii/S0021999102970713

[25] A. Suzuki, T. Shigeyama, Detailed analysis of filamentary structure in the Weibel instability, The Astrophysical Journal 695 (2) (2009) 1550.

URL http://stacks.iop.org/0004-637X/695/i=2/a=1550

[26] A. Ghizzo, P. Bertrand, M. Shoucri, E. Fijalkow, M. Feix, An eulerian code for the study of the drift-kinetic vlasov equation, Journal of Computational Physics 108 (1) (1993) 105 - 121. doi:http://dx.doi.org/10.1006/jcph.1993.1167.

URL http://www.sciencedirect.com/science/article/pii/S0021999183711678

[27] E. Sonnendrücker, J. Roche, P. Bertrand, A. Ghizzo, The semi-Lagrangian method for the numerical resolution of the Vlasov equation, Journal of Computational Physics 149 (2) (1999) 201-220. doi:http://dx.doi.org/10.1006/jcph.1998.6148.

URL http://www.sciencedirect.com/science/article/pii/S0021999198961484

[28] R. A. Dory, G. E. Guest, E. G. Harris, Unstable electrostatic plasma waves propagating perpendicular to a magnetic field, Phys. Rev. Lett. 14 (1965) 131-133. doi:10.1103/PhysRevLett.14.131.

URL http://link.aps.org/doi/10.1103/PhysRevLett.14.131

[29] J. A. Byers, M. Grewal, Perpendicularly propagating plasma cyclotron instabilities simulated with a one-dimensional computer model, Physics of Fluids 13 (7) (1970) 1819-1830. doi:http://dx.doi.org/10.1063/1.1693160.

URL http://scitation.aip.org/content/aip/journal/pof1/13/7/10.1063/1.1693160

[30] F. Crawford, J. Tataronis, Absolute instabilities of perpendicularly propagating cyclotron harmonic plasma waves, Journal of Applied Physics 36 (9) (1965) 2930-2934. doi:http://dx.doi.org/10.1063/1.1714609.

URL http://scitation.aip.org/content/aip/journal/jap/36/9/10.1063/1.1714609

[31] J. Tataronis, F. Crawford, Cyclotron harmonic wave propagation and instabilities: I. perpendicular 
propagation, Journal of Plasma Physics 4 (1970) 231-248. doi:10.1017/S0022377800004979.

URL http://journals . cambridge.org/article_S0022377800004979

[32] I. B. Bernstein, Waves in a plasma in a magnetic field, Physical Review 109 (1) (1958) 10-21. doi:10.1103/PhysRev.109.10.

URL http://link.aps.org/doi/10.1103/PhysRev.109.10

[33] N. Krall, A. Trivelpiece, Principles of Plasma Physics, McGraw-Hill, New York, NY, 1973.

[34] D. A. Gurnett, A. Bhattacharjee, Introduction to plasma physics: with space and laboratory applications, Cambridge University Press, 2005.

[35] F. Crawford, A review of cyclotron harmonic phenomena in plasmas, Nuclear Fusion 5 (1) (1965) 73. doi:10.1088/0029-5515/5/1/010.

URL http://stacks.iop.org/0029-5515/5/i=1/a=010

[36] G. Cottrell, R. Dendy, Superthermal radiation from fusion products in JET, Physical Review Letters 60 (1) (1988) 33-36. doi:10.1103/PhysRevLett.60.33.

URL http://link.aps.org/doi/10.1103/PhysRevLett.60.33

[37] A. Goede, P. Massmann, H. Hopman, J. Kistemaker, Ion Bernstein waves excited by an energeticion beam ion a plasma, Nuclear Fusion 16 (1) (1976) 85. doi:10.1088/0029-5515/16/1/009.

URL http://stacks.iop.org/0029-5515/16/i=1/a=009

[38] R. F. Hubbard, T. J. Birmingham, Electrostatic emissions between electron gyroharmonics in the outer magnetosphere, Journal of Geophysical Research: Space Physics 83 (A10) (1978) 4837-4850. doi:10.1029/JA083iA10p04837.

URL http://dx.doi.org/10.1029/JA083iA10p04837

[39] S. Perraut, A. Roux, P. Robert, R. Gendrin, J.-A. Sauvaud, J.-M. Bosqued, G. Kremser, A. Korth, A systematic study of ULF waves above FH+ from GEOS 1 and 2 measurements and their relationships with proton ring distributions, Journal of Geophysical Research: Space Physics 87 (A8) (1982) 62196236. doi:10.1029/JA087iA08p06219.

URL http://dx.doi.org/10.1029/JA087iA08p06219

[40] M. Ashour-Abdalla, C. Kennel, Nonconvective and convective electron cyclotron harmonic instabilities, Journal of Geophysical Research: Space Physics 83 (A4) (1978) 1531-1543. doi:10.1029/JA083iA04p01531.

URL http://dx.doi.org/10.1029/JA083iA04p01531

[41] R. L. Kaufmann, P. B. Dusenbery, B. J. Thomas, Stability of the auroral plasma: Parallel and perpendicular propagation of electrostatic waves, Journal of Geophysical Research: Space Physics 83 (A12) (1978) 5663-5669. doi:10.1029/JA083iA12p05663.

URL http://dx.doi.org/10.1029/JA083iA12p05663

[42] R. F. Post, M. Rosenbluth, Electrostatic instabilities in finite mirror-confined plasmas, Physics of Fluids 9 (4) (1966) 730-749. doi:http://dx.doi.org/10.1063/1.1761740.

URL http://scitation.aip.org/content/aip/journal/pof1/9/4/10.1063/1.1761740

[43] G. Guest, Electron Cyclotron Heating of Plasmas, John Wiley and Sons, 2009.

[44] E. Harris, Plasma instabilities associated with anisotropic velocity distributions, Journal of Nuclear Energy. Part C, Plasma Physics, Accelerators, Thermonuclear Research 2 (1) (1961) 138. doi:10.1088/0368$3281 / 2 / 1 / 322$.

URL http://stacks.iop.org/0368-3281/2/i=1/a=322

[45] J. K. Lee, C. K. Birdsall, Velocity space ring-plasma instability, magnetized, part ii: Simulation, Physics of Fluids 22 (7) (1979) 1315-1322. doi:http://dx.doi.org/10.1063/1.862734. URL http://scitation.aip.org/content/aip/journal/pof1/22/7/10.1063/1.862734

[46] T. Umeda, M. Ashour-Abdalla, D. Schriver, R. L. Richard, F. V. Coroniti, Particle-in-cell simulation of Maxwellian ring velocity distribution, Journal of Geophysical Research: Space Physics 112 (A4). doi:10.1029/2006JA012124. URL http://dx.doi.org/10.1029/2006JA012124

[47] P. Colella, M. Dorr, J. Hittinger, D. Martin, High-order, finite-volume methods in mapped coordinates, Journal of Computational Physics 230 (8) (2011) 2952 - 2976. 
doi:http://dx.doi.org/10.1016/j.jcp.2010.12.044.

URL http://www.sciencedirect.com/science/article/pii/S0021999111000064

[48] C.-W. Shu, Essentially non-oscillatory and weighted essentially non-oscillatory schemes for hyperbolic conservation laws, in: A. Quarteroni (Ed.), Advanced Numerical Approximation of Nonlinear Hyperbolic Equations, Vol. 1697 of Lecture Notes in Mathematics, Springer Berlin Heidelberg, 1998, pp. 325-432. doi:10.1007/BFb0096355.

URL http://dx.doi.org/10.1007/BFb0096355

[49] E. Harris, Unstable plasma oscillations in a magnetic field, Phys. Rev. Letters 2 (2) (1959) 34-36. doi:10.1103/PhysRevLett.2.34.

URL http://link.aps.org/doi/10.1103/PhysRevLett.2.34

[50] A. Erdélyi, W. Magnus, F. Oberhettinger, F. G. Tricomi, H. Bateman, Higher transcendental functions (California Institute of Technology H. Bateman MS Project), Vol. 2, McGraw-Hill, New York, NY, 1953.

URL http://apps.nrbook.com/bateman/Vol2.pdf

[51] F. Crawford, Cyclotron harmonic waves in warm plasmas, Journal of Research of the National Bureau of Standards 69D (6) (1965) 789.

URL http://archive.org/details/jresv69Dn6p789 